\title{
SUBGROUPS GENERATED BY TWO PSEUDO-ANOSOV ELEMENTS IN A MAPPING CLASS GROUP. II. UNIFORM BOUND ON EXPONENTS
}

\author{
KOJI FUJIWARA
}

In memory of Professor Shoshichi Kobayashi

\begin{abstract}
Let $S$ be a compact orientable surface, and $\operatorname{Mod}(S)$ its mapping class group. Then there exists a constant $M(S)$, which depends on $S$, with the following property. Suppose $a, b \in \operatorname{Mod}(S)$ are independent (i.e., $\left[a^{n}, b^{m}\right] \neq 1$ for any $n, m \neq 0$ ) pseudo-Anosov elements. Then for any $n, m \geq M$, the subgroup $\left\langle a^{n}, b^{m}\right\rangle$ is a free group freely generated by $a^{n}$ and $b^{m}$, and convexcocompact in the sense of Farb-Mosher. In particular all non-trivial elements in $\left\langle a^{n}, b^{m}\right\rangle$ are pseudo-Anosov. We also show that there exists a constant $N$, which depends on $a, b$, such that $\left\langle a^{n}, b^{m}\right\rangle$ is a free group freely generated by $a^{n}$ and $b^{m}$, and convex-cocompact if $|n|+|m| \geq N$ and $n m \neq 0$.
\end{abstract}

\section{INTRODUCTION}

Many groups $G$ are known to satisfy the Tits alternative; namely, each subgroup in $G$ contains a free group of rank two unless it is virtually solvable. A typical argument is to find "independent" elements $a$ and $b$ in the subgroup, then show $a^{n}$ and $b^{m}$ freely generate a free group of rank two for sufficiently large $n, m$. This argument applies to, for example, word-hyperbolic groups and also mapping class groups. The main goal of this paper is to obtain an upper bound on $n, m$ which does not depend on $a, b$, but only on the group $G$. We discuss groups $G$ acting on $\delta$-hyperbolic spaces by isometries.

This paper is an improvement on [7, but one can read this paper independently. We explain the improvement after we state the main results in this section.

1.1. Hyperbolic isometry and quasi-isometric embedding. A geodesic space is called $\delta$-hyperbolic for $\delta \geq 0$ if for any geodesics $\alpha, \beta, \gamma$ which form a triangle, $\alpha$ is contained in the $\delta$-neighborhood of $\beta \cup \gamma([8])$. Let $\Gamma$ be a $\delta$-hyperbolic space and $a$ an isometry of $\Gamma$. If there exist a point $x \in \Gamma$ and a constant $C>0$ such that $d\left(x, a^{n}(x)\right) \geq C n$ for any $n>0$, then $a$ is called hyperbolic.

Suppose that a finitely generated group $G$ is acting on $\Gamma$ by isometries. Fix a finite generating set and let $|a|$ be the word length of $a \in G$. Let $x \in \Gamma$ be a point and consider the map from $G$ to $\Gamma$ defined by sending $a \in G$ to $a(x) \in \Gamma$. We call

Received by the editors September 13, 2009 and, in revised form, May 1, 2012, September 5, 2013, and September 28, 2013.

2010 Mathematics Subject Classification. Primary 20F65; Secondary 20F67, 20F38, 20 F28. 
this map the embedding by an orbit of the action by $G$. If there exist constants $L, C>0$ such that for all $a \in G$

$$
|a| / L-C \leq d(x, a(x)) \leq L|a|+C,
$$

then we say the map is a quasi-isometric embedding.

Notice that since $G$ is finitely generated, the embedding by an orbit is always Lipschitz; therefore, only a lower bound is important. Also, whether the embedding is a quasi-isometric or not does not depend on the choice of the base point $x$, nor on the choice of the generating set of $G$.

1.2. Main result. The main result is the following. The point is that although the constant $M$ depends on $\Gamma$ and the action of $G$ on $\Gamma$, it does not depend on $a$ and $b$. It will become clear how the constant $M$ depends on the action.

Theorem 23. Suppose a group $G$ acts acylindrically on a $\delta$-hyperbolic graph $\Gamma$ by isometries. Then there exists a constant $M$ with the following property. Suppose $a, b \in G$ act hyperbolically. Assume for any $p, q \neq 0,\left[a^{p}, b^{q}\right] \neq 1$ in $G$. Then for any $n, m \geq M,\left\langle a^{n}, b^{m}\right\rangle$ is a free group freely generated by $a^{n}, b^{m}$. Moreover, the embedding of the free group $\left\langle a^{n}, b^{m}\right\rangle$ by an orbit in $\Gamma$ is quasi-isometric. In particular, all non-trivial elements in $\left\langle a^{n}, b^{m}\right\rangle$ are hyperbolic on $\Gamma$.

The acylindricity of an action (see section 3.3 for the definition) is a weak assumption on properness, and in particular, the result applies to a word-hyperbolic group and its action on a Cayley graph (see Corollary 24).

1.3. Convex cocompact subgroup in $\operatorname{Mod}(S)$. Our main application is regarding subgroups of mapping class groups. Let $S$ be a compact orientable surface, possibly with boundary, and $\operatorname{Mod}(S)$ its mapping class group (this is the group of isotopy classes of orientation preserving homeomorphisms $S \rightarrow S$. We do not assume its identity on $\partial S)$. Let $\mathcal{C}(S)$ be the curve graph of $S$ on which $\operatorname{Mod}(S)$ acts by isometries (see for example 13, 19, 3 for the definition).

Masur-Minsky 19 showed that $\mathcal{C}(S)$ is $\delta$-hyperbolic and an element $a \in \operatorname{Mod}(S)$ is pseudo-Anosov if and only if it acts as a hyperbolic isometry on $\mathcal{C}(S)$.

For a subgroup $G<\operatorname{Mod}(S)$, Farb-Mosher [6] introduced the notion of convexcocompact in terms of the action on Teichmüller space. It has been shown ([9], [15]) that $G$ is convex-cocompact if and only if the embedding of $G$ by an orbit in $\mathcal{C}(S)$ is quasi-isometric.

Since the action of $\operatorname{Mod}(S)$ on $\mathcal{C}(S)$ is acylindrical, our theorem applies. Namely, we show the following in Section 4 .

Theorem 27, Let $S$ be a compact orientable surface, and $\operatorname{Mod}(S)$ its mapping class group. Then there exists a constant $M(S)$ with the following property. Suppose $a, b \in \operatorname{Mod}(S)$ are pseudo-Anosov elements such that $\left[a^{n}, b^{m}\right] \neq 1$ for any $n, m \neq 0$ ( $a, b$ are called independent). Then for any $n, m \geq M$, the subgroup $\left\langle a^{n}, b^{m}\right\rangle$ is a free group freely generated by $a^{n}, b^{m}$ and convex-cocompact in the sense of FarbMosher. In particular all non-trivial elements in $\left\langle a^{n}, b^{m}\right\rangle$ are pseudo-Anosov.

If $S$ is a 2-sphere minus at most three points, then $M C G(S)$ is virtually abelian and elements $a, b$ which satisfy the assumption do not exist. If $S$ is a 2 -sphere minus four points or a 2-torus minus at most one point, then $M C G(S)$ is virtually free (and the theorem applies). 
It was known ([13, 20] ) that $\left\langle a^{n}, b^{m}\right\rangle$ is free for sufficiently large $n, m$. A uniform bound on $n, m$ is new. In our previous study 7, a uniform bound on one of $n, m$ was shown. Namely, there exists a constant $N(S)$ such that $\left\langle a^{n}, b^{m}\right\rangle$ is free and convex-cocompact if one of $n, m$ is at least $N$ and the other one is sufficiently large. The previous study has also been used by Mangahas [18] to show the uniform exponential growth of a mapping class group.

We do not know a bound for $M(S)$. This is because our proof uses the acylindricity of Bowditch 3. This property is quantified by sets of numbers $K(R), L(R)$ (see Definition 10), and we need estimates (at least at certain values for $R$ ) on those to have a bound on $M(S)$, but no estimates are known. All other constants are explicit in the argument. It would be interesting to know the asymptotics of $M(S)$ in terms of the Euler number of $S$.

Our result concerns only pseudo-Anosov elements $a, b$. It is unknown if a uniform bound such as $M(S)$ exists for two elements $a, b$ of infinite order in general such that the subgroup $\left\langle a^{n}, b^{m}\right\rangle$ is free if $n, m \geq M$. (Note that if $a$ and $b$ have infinite order, the subgroup is never convex-cocompact unless both $a$ and $b$ are pseudoAnosov.) In the case that both $a, b$ are Dehn twists ([1]), and more generally, positive multi-twists ([10, Theorem 3.2]), it is known that $\left\langle a^{n}, b^{m}\right\rangle$ is a free group of rank two (but not convex-cocompact) or abelian if $n, m \geq 2$. Recently, Leininger and Margalit [17] have shown that if $S$ is the $n$-times punctured sphere, then for any two elements $a, b \in \operatorname{Mod}(S),\left\langle a^{N}, b^{N}\right\rangle$ is either a free group of rank two or abelian for $N=n$ !.

It would be interesting to know for which $(n, m)$, the subgroup $\left\langle a^{n}, b^{m}\right\rangle$ is a free group of rank two for given $a, b$ in our setting. The following theorem says that for given $a, b$, the subgroup $\left\langle a^{n}, b^{m}\right\rangle$ is free except for finitely many pairs $(n, m)$. It is not clear if the number of those exceptional pairs $(n, m)$ is bounded, but the constant $N$ must depend on $a, b$ in the following theorem (see Example 29).

Theorem 28, Let $S$ be a compact orientable surface and $a, b$ two independent pseudo-Anosov elements. Then there exists $N$ such that for any $n \geq N$, both $\left\langle a, b^{n}\right\rangle$ and $\left\langle b, a^{n}\right\rangle$ are free groups freely generated by the two elements and are convex-cocompact. In particular, $\left\langle a^{n}, b^{m}\right\rangle$ is a free group freely generated by the two elements and is convex-cocompact if $|n|+|m| \geq 2 N$ and $n m \neq 0$.

In this paper we work out constants (not best constants) in various arguments. We also give arguments for some versions of standard facts in the appendix. We hope it will be helpful in other places. The author thanks the referee, who suggested this idea.

\section{Preliminaries}

We collect basic definitions and properties. Let $X$ be a metric space. For two points $x, y \in X$, we write the distance between the two points by $|x-y|$.

2.1. Paths, subpaths and the order on points. Let $I$ be a connected interval. Maybe it is infinite or bi-infinite. A path is a map $\gamma: I \rightarrow X$. In this paper, we only consider paths with the maps continuous. We may identify the map and its image. For points $p=\gamma(u), q=\gamma(v)$ with $u \leq v$ on the path $\gamma$, define the subpath between $p$ and $q$ as

$$
\gamma_{p, q}=\{\gamma(t) \mid u \leq t \leq v\}
$$


Define it similarly if $u>v$. If $\gamma$ is not injective, there is an ambiguity since $\gamma_{p, q}$ actually depends on the parameters $u, v$. So when we use this notation, if needed we specify the parameters $u, v$. We will see that any two choices of $\gamma_{p, q}$ are in a bounded neighborhood of each other if $\gamma$ is a quasi-axis (see the remark before the proof of Lemma 10. We say a point in $\gamma_{p, q}$ is between $p$ and $q$ (we use this expression when the parameters $u, v$ are understood). For points $p=\gamma(u), q=\gamma(v)$ with $u<v$, we sometimes write $p<q$. We may just write $p<q$ if there exist such $u<v$ for $p, q$.

2.2. Axis and quasi-axis. Let $\Gamma$ be a $\delta$-hyperbolic space. For $x, y \in \Gamma$, let $[x, y]$ be a geodesic from $x$ to $y$. The geodesic is maybe not unique.

Many arguments in the paper will be estimates of constants using $\delta$-hyperbolic geometry. There will be two important constants:

$$
\Delta=418 \delta, \Lambda=2 \Delta+3 \delta .
$$

They are chosen carefully so that the argument works. If $\delta=0$, then they are 0 .

Suppose $a$ is a hyperbolic isometry of $\Gamma$. If there exists a geodesic $\alpha$ such that $a(\alpha)=\alpha$, then $\alpha$ is called an axis of $a$. Axes do not always exist in general.

The following fact is by elementary $\delta$-hyperbolic geometry, but we do not know any reference to it in this form, and we give an argument in the appendix with $\Delta=418 \delta$. We said there is an ambiguity in the definition of $\alpha_{p, q}$, but the lemma holds for any subpath between $p$ and $q$ (see the end of the proof of the lemma in the appendix).

Lemma 1 (Quasi-axis). If a is a hyperbolic isometry of a $\delta$-hyperbolic space $\Gamma$, there exists a path $\alpha$ such that

(1) $a(\alpha)=\alpha$ and there exists $L>0$ such that $a(\alpha(t))=\alpha(t+L)$.

(2) Let $p, q \in \alpha$. Then a geodesic $[p, q]$ and a subpath $\alpha_{p, q}$ between $p, q$ are in the $\Delta$-neighborhood of each other.

$\Delta$ depends only on $\delta . \Delta=0$ if $\delta=0$.

We call such a path $\alpha$ a quasi-axis of $a$ in this paper. We take the parametrization $t$ of $\alpha$ by arc length such that $L>0$. This is the parametrization we use when we write $p(u)<q(v)$ for points on $\alpha$ if $u<v$. Note that for any point $p \in \alpha$, we have $p<a(p)$, since if $p=\alpha(u)$, then the parameter of $a(p)$ is $u+L$.

A quasi-isometric map from a connected interval is called quasi-geodesic. We may also assume that a quasi-axis is a quasi-geodesic, but we do not use this fact. For us, (2) is more important. It follows from (1) and (2) that any two quasi-axes of $a$ are in the $(2 \Delta+2 \delta)$-neighborhood of each other.

Of course an axis is a quasi-axis. The arguments become simpler if there are axes for hyperbolic elements we discuss, but quasi-axes are always enough for us. We only need to deal with constants which occur as error terms in the argument.

There is a notion of the ideal boundary $\partial_{\infty} \Gamma$ of a $\delta$-hyperbolic space $\Gamma$ ([8]). A quasi-axis of a hyperbolic isometry $g$ defines two points in the ideal boundary, and $g$ has north-south dynamics with those two points fixed. Regarding Theorem 23, one can prove $\left\langle a^{n}, b^{m}\right\rangle$ is free if $n, m$ are sufficiently large using the dynamics on $\partial_{\infty} \Gamma$, but we do not know how to get a uniform bound on $n, m$ by this approach.

2.3. Translation length. For an isometry $a$ of $X$, we define its translation length, $\operatorname{tr}(a)$, by

$$
\operatorname{tr}(a)=\liminf _{n \rightarrow \infty} \frac{\left|x-a^{n}(x)\right|}{n} \geq 0
$$


for a point $x$. By triangle inequality, $\operatorname{tr}(a)$ does not depend on the choice of $x$. Since the sequence $\left\{\left|x-a^{n}(x)\right|\right\}_{n \geq 1}$ is subadditive by triangle inequality, in fact $\lim$ inf $=\inf =\lim$ in the above definition by Fekete's subadditive lemma (cf. [21]), and it follows that $\operatorname{tr}\left(a^{n}\right)=|n| \operatorname{tr}(a)$ for any $n$. The isometry $a$ is hyperbolic iff $\operatorname{tr}(a)>0$.

Lemma 2. If a is a hyperbolic isometry on a $\delta$-hyperbolic space with a quasi-axis $\alpha$, then for any $N>0$ and any point $p \in \alpha$, we have

$$
\left|p-a^{N}(p)\right|-2 \delta-2 \Delta \leq N \operatorname{tr}(a) \leq\left|p-a^{N}(p)\right| .
$$

We will give a proof in the appendix.

2.4. $C$-intersection. For a subset $A \subset \Gamma$, let $N_{C}(A)$ be the $C$-neighborhood of $A$. Sometimes, $A_{C}$ will denote the $C$-neighborhood of the set $A$ as well.

Definition 3. We define the $C$-intersection of two subsets $A$ and $B$, denoted by $A \cap_{C} B$, as follows:

$$
A \cap_{C} B=\left(A \cap N_{C}(B)\right) \cup\left(B \cap N_{C}(A)\right) .
$$

We define

$$
\left|A \cap_{C} B\right|=\max \left(\operatorname{diam}\left(A \cap N_{C}(B)\right), \operatorname{diam}\left(B \cap N_{C}(A)\right)\right),
$$

which is maybe $\infty$. The diameter of an empty set is defined to be 0 .

For three numbers $K, L, M$, if $|K-L| \leq M$, then we write $K \sim_{M} L$.

Clearly, $\operatorname{diam}\left(A \cap N_{C}(B)\right) \sim_{2 C} \operatorname{diam}\left(B \cap N_{C}(A)\right) \sim_{2 C}\left|A \cap_{C} B\right|$.

For quasi-axes $\alpha, \beta$, if $\alpha \cap_{C} \beta$ is non-empty, then this subset looks like a narrow tube. In fact, if the set is bounded, then it is contained in $N_{C+2 \Delta+2 \delta}([P, Q])$, where $P, Q \in \alpha$ are first/last points in $\alpha \cap N_{C}(\beta)$. We call those $P, Q$ extremal points in $\alpha$. To prove this fact, we need a lemma.

Lemma 4. Let $A, B, C, D$ be points in $\delta$-hyperbolic space with $|A-C|,|B-D| \leq L$. Then $A D \subset C D_{L+2 \delta}$.

Proof. We have $A B \subset\left(A D_{\delta} \cup B D_{\delta}\right), B D \subset C D_{L}$, and $A D \subset C D_{L+\delta}$. It follows that $A D \subset C D_{L+2 \delta}$.

Now, let $P^{\prime}, Q^{\prime} \in \beta$ be the extremal points. Notice that $P^{\prime}, Q^{\prime} \in[P, Q]_{\Delta+C}$ by the definition of extremal points and Lemma 1 (2). Again by the same lemma, $\alpha \cap_{C} \beta \subset[P, Q]_{\Delta} \cup\left[P^{\prime}, Q^{\prime}\right]_{\Delta} \subset[P, Q]_{C+2 \Delta+2 \delta}$. We used Lemma 4 for the last inclusion.

Also, by Lemma 1 (2), $\operatorname{diam}\left(\alpha \cap N_{C}(\beta)\right) \sim_{2 \Delta}|P-Q|$; therefore, $|P-Q| \sim_{2 \Delta+2 C}$ $\left|\alpha \cap_{C} \beta\right|$.

We state a lemma we use later.

Lemma 5. For points $x, y, z$ in a $\delta$-hyperbolic space, we have

$$
|x-y|+|x-z|-|y-z| \sim_{6 \delta} 2\left|[x, y] \cap_{\delta}[x, z]\right| .
$$

Proof. Move a point from $x$ to $y$ on $[x, y]$, and let $p$ be the last point with $p \in$ $N_{\delta}([x, z])$. (Maybe $p=y$.) Then $p \in N_{\delta}([y, z])$. Let $q \in[x, z]$ and $r \in[y, z]$ be such that $|q-p|,|r-p| \leq \delta$. Then $|q-r| \leq 2 \delta$.

Now, combine $|x-p| \sim_{\delta}\left|[x, y] \cap_{\delta}[x, z]\right|$ and $|x-y|+|x-z|-|y-z| \sim_{4 \delta} 2|x-p|$. 
For points $a, b, c$, define

$$
(a, b)_{c}=\frac{|a-c|+|b-c|-|a-b|}{2} .
$$

This is called the Gromov product of $a, b$ w.r.t. c. We rephrase Lemma 5 as follows.

Lemma 6. If $X$ is $\delta$-hyperbolic, then we have the following for any $a, b, c \in X$ :

$$
\left|[a, c] \cap_{\delta}[b, c]\right| \sim_{3 \delta}(a, b)_{c} .
$$

\section{Generating free subgroups}

3.1. Overview. In this section, we will find sufficient conditions for certain powers of two independent hyperbolic isometries $a, b$ to freely generate a free group. The final result is Theorem 23 .

It is well-known that for sufficiently large $n, m>0, a^{n}, b^{m}$ generate a free group (Proposition 8). The argument is an application of a geometric fact on a $\delta$-hyperbolic space (Proposition 7 ). We will give an upper bound on $n, m$ which does not depend on $a$ and $b$. Since we use the idea repeatedly, we give details of the argument for Proposition 8 ,

In Section 3.3 , by analyzing the argument for Proposition 8 carefully, we first show that there is an upper bound on $n$ and $m$ if the translation lengths of $a$ and $b$ are comparable (Proposition 17). One extra issue is that we do not assume that the action is proper, but only acylindrical in view of the application to mapping class groups.

The other case, namely, one of the translation lengths, say for $b$, is much smaller than the translation length of $a$, needs a new idea. In Section 3.4, we deal with this case and show there is an upper bound on both $n$ and $m$ in this case too (Proposition 18). In fact we can always take $m=1$. This proposition is novel.

The two propositions are complimentary to each other, and combining them, we obtain an upper bound on both exponents which does not depend on $a$ and $b$ in Section 3.5 (Theorem 23).

3.2. Nielsen condition. We review a well-known fact (Proposition 8) and its proof. We start with a fundamental result by Gromov [8, 7.2 C]. We quote it from [4, Lemma 1.1] (see also [5, Lemma 1.3.4]).

Proposition 7 (Three points condition). Let $\Gamma$ be a $\delta$-hyperbolic space. Let $p_{i} \in$ $\Gamma(i \geq 1)$ be a sequence of points and $U>0$ a constant such that for all $i \geq 1$,

$$
\left|p_{i}-p_{i+2}\right| \geq \max \left(\left|p_{i}-p_{i+1}\right|,\left|p_{i+1}-p_{i+2}\right|\right)+2 \delta+U .
$$

Then, for all $i, j \geq 1$,

$$
\left|p_{i}-p_{j}\right| \geq U|i-j| \text {. }
$$

We call the inequality (11) the three points condition (for $p_{i}, p_{i+1}, p_{i+2}$ and a constant $U$ ). If the three points condition is satisfied for any three consecutive points in a sequence, then we say the sequence satisfies the three points condition. Note that the three points condition for $P, Q, R$ is equivalent to

$$
|P-Q|+|Q-R|-|P-R|+(U+2 \delta) \leq \min (|P-Q|,|Q-R|) .
$$

Usually we try to verify this in the argument. 
Various versions of the following proposition are well-known ([8]; see also [16]), but we give a detailed proof of this version, since we will generalize the statement (and the argument).

Let

$$
\Lambda=2 \Delta+3 \delta .
$$

If $\delta=0$, then both $\Delta, \Lambda=0$. In the rest of the paper, the subset $\alpha \cap_{\Lambda} \beta$ for quasi-axes $\alpha, \beta$ is important.

Proposition 8 (Nielsen condition). Suppose isometries $a, b$ act hyperbolically on a $\delta$-hyperbolic space $\Gamma$ with quasi-axes $\alpha, \beta$. If $1 \leq n, m \in \mathbb{Z}$ are such that for some constant $U>0$,

$\operatorname{tr}\left(a^{n}\right) \geq\left|\alpha \cap_{\Lambda} \beta\right|+26 \delta+14 \Delta+4 \Lambda+2 U, \operatorname{tr}\left(b^{m}\right) \geq\left|\alpha \cap_{\Lambda} \beta\right|+26 \delta+14 \Delta+4 \Lambda+2 U$, then $\left\langle a^{n}, b^{m}\right\rangle<\operatorname{Isom}(\Gamma)$ is a free group freely generated by $a^{n}, b^{m}$. Moreover, the embedding of the subgroup $\left\langle a^{n}, b^{m}\right\rangle$ to $\Gamma$ by an orbit is quasi-isometric.

Proof. Set $A=a^{n}, B=b^{m}$. We will find a point $p \in \Gamma$ such that for any non-trivial reduced word $w$ on $A, B$,

$$
U|w| \leq|w(p)-p|
$$

where $|w|$ is the word length of $w$ with respect to $A, B$. This implies that $A, B$ freely generate a rank two free group, and the embedding of $\langle A, B\rangle$ in $\Gamma$ using the orbit of $p$ is bi-Lipschitz.

There are two cases: (i) $\alpha \cap_{\Lambda} \beta \neq \emptyset$, (ii) $\alpha \cap_{\Lambda} \beta=\emptyset$. We first discuss (i) in detail.

Let $S, T \in \alpha$ be the extremal points for $\alpha \cap_{\Lambda} \beta$, and $Q$ the mid-point of $[S, T]=\ell$. Take $p \in \alpha$ with $|p-Q| \leq \Delta$. Then $p \in N_{2 \delta+2 \Delta+\Lambda}(\beta)$.

Suppose

$$
w=A^{n_{1}} B^{m_{1}} \cdots A^{n_{i}} B^{m_{i}}
$$

where $n_{1}, m_{i}$ are possibly 0 . We discuss the case such that both $n_{1}, m_{i}$ are not 0 (the other cases are similar and we omit them). To use Proposition 7 , we define a sequence of points in $\Gamma$ from $p$ to $w(p)$. Let $\Pi$ be the Cayley graph of the free group $\langle A, B\rangle$ freely generated by $A, B$. Here we abuse letters $A, B$ to define the free group. $\Pi$ is a tree. In $\Pi$, on the geodesic from 1 to $w$, the following vertices appear in this order:

$$
1, A, A^{2}, \cdots, A^{n_{1}}, A^{n_{1}} B, A^{n_{1}} B^{2}, \cdots, A^{n_{1}} \cdots A^{n_{i}} B^{m_{i}-1}, w
$$

if $n_{1}, m_{1}, m_{i}>0$. For example, if $n_{1}<0$, then it will be

$$
1, A^{-1}, A^{-2}, \cdots, A^{n_{1}}, \cdots, w .
$$

For notational convenience, we will usually assume in this paper that the exponents are positive when we list elements. We will say so when we do that.

The sequence of points in $\Gamma$ will be the image of $p$ by those elements in $\langle A, B\rangle$ in this order. We label them as $p_{j}$ or $p_{j, k}$ in the following way. First define

$$
\begin{aligned}
p_{0} & =p, p_{1}=A^{n_{1}}(p), p_{2}=A^{n_{1}} B^{m_{1}}(p), p_{3}=A^{n_{1}} B^{m_{1}} A^{n_{2}}(p), \cdots, \\
p_{2 i-1} & =A^{n_{1}} B^{m_{1}} \cdots A^{n_{i}}(p), p_{2 i}=A^{n_{1}} B^{m_{1}} \cdots A^{n_{i}} B^{m_{i}}(p),
\end{aligned}
$$

then define more points between $p_{0}$ and $p_{1}$ :

$$
p_{0,0}=p_{0}, p_{0,1}=A(p), p_{0,2}=A^{2}(p), \cdots, p_{0, n_{1}}=A^{n_{1}}(p)=p_{1},
$$

and between $p_{1}$ and $p_{2}$ :

$$
p_{1,0}=p_{1}, p_{1,1}=A^{n_{1}} B(p), p_{1,2}=A^{n_{1}} B^{2}(p), \cdots, p_{1, m_{1}}=A^{n_{1}} B^{m_{1}}(p)=p_{2} .
$$


Similarly, define additional points between $p_{2 j}$ and $p_{2 j+1}$, and also between $p_{2 j}$ and $p_{2 j-1}$ for all $j$. We obtain a sequence of points $p_{j, k}$ with the order (the lexicographical order on $(j, k))$.

We claim that the sequence $\left\{p_{j, k}\right\}$ satisfies the three points condition for $U$. It then follows from Proposition 7 that

$$
\left|p_{2 i}-p_{0}\right| \geq U\left(\left|n_{1}\right|+\left|m_{1}\right|+\cdots+\left|n_{i}\right|+\left|m_{i}\right|\right)
$$

since there are $\left|n_{1}\right|+\left|m_{1}\right|+\cdots+\left|n_{i}\right|+\left|m_{i}\right|+1$ points between $p_{0}$ and $p_{2 i}$ including the two points. We are done since $|w|=\left|n_{1}\right|+\left|m_{1}\right|+\cdots+\left|n_{i}\right|+\left|m_{i}\right|$ and $p_{2 i}=$ $w(p), p_{0}=p$.

We now verify the three points condition. There are two cases and the easy one is such that the three (consecutive) points, say, $x, y, z$, are between $p_{j}$ and $p_{j+1}$ for some $j$. To be concrete, suppose that $x, y, z$ is (a) between $p_{0}$ and $p_{1}$ or (b) between $p_{1}$ and $p_{2}$. Then, the three points are on the quasi-axis $\alpha$ in (a) or "almost" (since $p$ is not exactly on $\beta$ ) on $A^{n_{1}}(\beta)$ in (b). We remark that $A^{n_{1}}(\beta)$ is a quasi-axis for $A^{n_{1}} B A^{-n_{1}}$.

For (a), it suffices to verify the three points condition for $A^{-1}(p), p, A(p) \in \alpha$. Since $\alpha$ is a quasi-axis, there is a point $P \in\left[A^{-1}(p), A(p)\right]$ with $|p-P| \leq \Delta$. Therefore, by the triangle inequality,

$$
\left|A^{-1}(p)-p\right|+|p-A(p)|-\left|A^{-1}(p)-A(p)\right| \leq 2 \Delta .
$$

By Lemma 2 $\left|A^{-1}(p)-p\right|=|p-A(p)| \geq \operatorname{tr}\left(a^{n}\right)-2 \delta-2 \Delta$; hence the three point condition is easy to verify (see the following discussion for (b)). The case (b) is similar. It suffices to verify the three points condition for $B^{-1}(p), p, B(p)$ after we apply $A^{-n_{1}}$ (and some power of $B$ ). The point $p$ is not exactly on $\beta$, but it is in the $(\Lambda+2 \delta+2 \Delta)$-neighborhood of $\beta$. It follows that

$\left|B^{-1}(p)-p\right|+|p-B(p)|-\left|B^{-1}(p)-B(p)\right| \leq(2 \Delta+6(\Lambda+2 \delta+2 \Delta))=12 \delta+14 \Delta+6 \Lambda$.

Also, $\left|B^{-1}(p)-p\right|=|p-B(p)| \geq \operatorname{tr}\left(b^{m}\right)-(2 \Delta+2 \delta)-2(\Lambda+2 \Delta+2 \delta)=\operatorname{tr}\left(b^{m}\right)-$ $6(\delta+\Delta)-2 \Lambda$. Combining those estimates and the original assumption, we have $\left|B^{-1}(p)-p\right|+|p-B(p)|-\left|B^{-1}(p)-B(p)\right|+(U+2 \delta) \leq U+14 \delta+14 \Delta+6 \Lambda \leq$ $\operatorname{tr}\left(b^{m}\right)-6(\delta+\Delta)-2 \Lambda \leq|p-B(p)|$. We are done with (a) and (b), and the argument for the first case is complete.

The other case is such that the second point is $p_{j}$ for some $j$. Again, to be concrete, look at the first such case, namely, $j=1$. Then the three points are

$$
A^{n_{1}-1}(p), A^{n_{1}}(p)=p_{1}, A^{n_{1}} B(p) .
$$

Apply $A^{-n_{1}}$ to those three points and obtain $A^{-1}(p), p, B(p)$. (If $n_{1}<0$, then we get $A(p), p, B(p)$.) It suffices to verify the condition for those three points.

We state a lemma.

\section{Lemma 9.}

$$
\left|p-A^{ \pm 1}(p)\right|+|p-B(p)|-\left|A^{ \pm 1}(p)-B(p)\right| \leq\left|\alpha \cap_{\Lambda} \beta\right|+22 \delta+14 \Delta+4 \Lambda .
$$

We postpone proving the lemma and continue. By the lemma, $\left|p-A^{ \pm 1}(p)\right|+$ $|p-B(p)|-\left|A^{ \pm 1}(p)-B(p)\right|+(U+2 \delta) \leq\left|\alpha \cap_{\Lambda} \beta\right|+24 \delta+14 \Delta+4 \Lambda+U$. By our assumption, this is $\leq \operatorname{tr}\left(a^{n}\right) \leq\left|p-A^{ \pm 1}(p)\right|$ and also $\leq \operatorname{tr}\left(b^{m}\right) \leq\left|p-B^{ \pm 1}(p)\right|$. The three points condition is shown. Now we prove the lemma. 
Proof. The argument is different for $A(p)$ and $A^{-1}(p)$. If $A$ maps $p$ to the same direction as $B$ does, then the left hand side of the desired inequality is larger for $A(p)$ than for $A^{-1}(p)$. So, in the proof, we assume that $A$ and $B$ map $p$ to the same direction and only prove the inequality for $A(p)$ (see Figure 1). The argument is easier for $A^{-1}(p)$ and we omit it. If $A^{-1}$ maps $p$ to the same direction as $B$, the argument is the same and we omit it.

Take $x \in[p, A(p)] \cap N_{\delta}[p, B(p)]$ with $|p-x| \geq\left|[p, A(p)] \cap_{\delta}[p, B(p)]\right|-2 \delta$. Take $y \in[p, B(p)]$ with $|x-y| \leq \delta$.

Since $\alpha$ is a quasi-axis and $x \in[p, A(p)]$, there exists $X \in \alpha$ with $|X-x| \leq \Delta$. Since $p, B(p) \in N_{\Lambda+2 \delta+2 \Delta}(\beta)$, it follows from $y \in[p, B(p)]$, unless (i) $|y-p| \leq \Lambda+$ $4 \delta+2 \Delta$ or (ii) $|y-B(p)| \leq \Lambda+4 \delta+2 \Delta$, that there exists $Y \in \beta$ with $|Y-y| \leq \Delta+2 \delta$. Indeed, let $p^{\prime}, p^{\prime \prime} \in \beta$ be points with $\left|p-p^{\prime}\right|,\left|B(p)-p^{\prime \prime}\right| \leq \Lambda+2 \delta+2 \Delta$. Then there is $y^{\prime} \in\left[p^{\prime}, p^{\prime \prime}\right]$ with $\left|y-y^{\prime}\right| \leq 2 \delta$ since both $|y-p|,|y-B(p)| \geq \Lambda+4 \delta+2 \Delta$. Now, use $y^{\prime} \in N_{\Delta}(\beta)$ and the triangle inequality. We discuss (i) and (ii) later.

We find that $|X-Y| \leq 2 \Delta+3 \delta=\Lambda$; therefore $X \in \alpha \cap_{\Lambda} \beta$; i.e., $X$ is contained in the subsegment of $\alpha$ between $S$ and $T$. So, since $T$ is an extremal point for $\alpha \cap_{\Lambda} \beta$, we have $|Q-X| \leq|Q-T|+\Delta$. (Remember that $Q$ is the mid-point of the two extremal points $S, T \in \alpha$.)

Now, by Lemma [5, $|p-A(p)|+|p-B(p)|-|A(p)-B(p)| \leq 2 \mid[p, A(p)] \cap_{\delta}$ $[p, B(p)]|+6 \delta \leq 2| p-x|+10 \delta \leq 2| Q-X|+10 \delta+4 \Delta \leq 2| Q-T \mid+2 \Delta+10 \delta+4 \Delta \leq$ $\operatorname{diam}\left(\alpha \cap N_{\Lambda}(\beta)\right)+2 \Lambda+10 \delta+6 \Delta \leq\left|\alpha \cap_{\Lambda} \beta\right|+2 \Lambda+10 \delta+6 \Delta$.

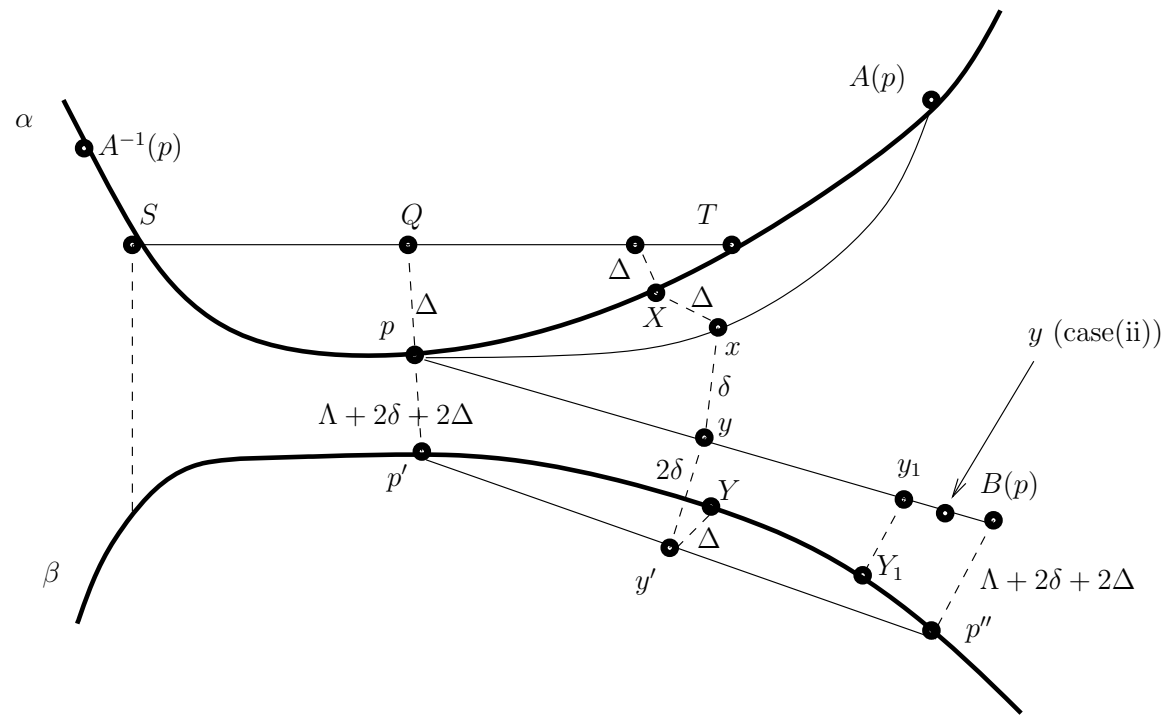

Figure 1. Lemma 9

We are left with the cases (i) and (ii). The case (i) is straightforward: $|p-A(p)|+$ $|p-B(p)|-|A(p)-B(p)| \leq 2|p-y|+12 \delta \leq 2(\Lambda+4 \delta+2 \Delta)+12 \delta=2 \Lambda+4 \Delta+20 \delta$. In case of (ii), maybe $X \notin \alpha \cap_{\Lambda} \beta$. Therefore we first retake the point $y$ to $y_{1} \in[p, B(p)]$ with $\left|y_{1}-B(p)\right|=\Lambda+4 \delta+2 \Delta$. Then $\left|y-y_{1}\right| \leq \Lambda+4 \delta+2 \Delta$ and $\left|y_{1}-p\right| \geq \Lambda+4 \delta+2 \Delta$. Accordingly, we (can) choose $Y_{1} \in \beta$ between $p^{\prime}$ and $p^{\prime \prime}$ with $\left|y_{1}-Y_{1}\right| \leq \Delta+2 \delta$, $x_{1} \in[p, A(p)]$ with $\left|y_{1}-x_{1}\right| \leq \delta$, and $X_{1} \in \alpha$ between $p, A(p)$ with $\left|X_{1}-x_{1}\right| \leq \Delta$. Then, $\left|X-X_{1}\right| \leq \Lambda+4 \Delta+6 \delta$. Also, $\left|X_{1}-Y_{1}\right| \leq 2 \Delta+3 \delta$; therefore $X_{1} \in \alpha \cap_{\Lambda} \beta$. 
Now, we argue in the same way with $X_{1}$ instead of $X$. At the end we get $|p-A(p)|+|p-B(p)|-|A(p)-B(p)| \leq\left|\alpha \cap_{\Lambda} \beta\right|+(2 \Lambda+10 \delta+6 \Delta)+2(\Lambda+6 \delta+4 \Delta)$. The error term is larger by $2(\Lambda+6 \delta+4 \Delta)$ since we retake $X$ to $X_{1}$. Lemma 9 is shown.

We are done with the case $\alpha \cap_{\Lambda} \beta \neq \emptyset$. Now we are left with the case such that $\alpha \cap_{\Lambda} \beta=\emptyset$. In this case, let $\ell$ be a geodesic segment which realizes the distance between $\alpha$ and $\beta$, and let $p \in \ell$ be the mid-point. Then the rest is the same as before, and verifying the three points condition is even easier. We omit details.

3.3. Acylindrical action. In this section, we assume certain properness of an action, acylindricity, and also improve Proposition 8 to Proposition 17, which has a bound on the exponents.

Definition 10 (Bowditch [3]). Let $\Gamma$ be a $\delta$-hyperbolic graph, and $G$ a group acting on $\Gamma$ by isometries. The action is acylindrical if for any $R>0$, there exist $K(R), L(R) \geq 1$ such that for any vertices $x, y \in \Gamma$ with $d(x, y) \geq L(R)$, the following set has at most $K(R)$ elements:

$$
\{g \in G \mid d(x, g(x)) \leq R, d(y, g(y)) \leq R\} .
$$

Remark 11. There is a notion called WPD (weak proper continuity), which is a priori weaker than acylindricity. WPD is defined for each hyperbolic isometry, and roughly speaking, the finiteness in the definition is required only for points $x$ and $y$ on a quasi-axis (see [1]). WPD is enough for some of our arguments.

The translation length is uniformly positive under acylindricity.

Lemma 12. Suppose $G$ acts on a $\delta$-hyperbolic space $\Gamma$. If the action is acylindrical with constants $K(R), L(R)$, then there exists an integer $P \geq 1$ such that for any element $a \in G$ which acts hyperbolically on $\Gamma$, we have $\operatorname{tr}\left(a^{P}\right) \geq 1$. The constant $P$ depends only on $\delta$ and $K(2(\delta+\Delta)+1)$.

Proof. Fix a constant $R>2(\delta+\Delta)$. (We will take $R=2(\delta+\Delta)+1$ at the end.) Let $\alpha$ be a quasi-axis of $a$. Fix a point $x \in \alpha$. Since $a$ is hyperbolic, let $N$ be such that $y=a^{N}(x) \in \alpha$ satisfies $|x-y| \geq L(R)$. We fix such $N$. For each $i \geq 0$, we have $\left|a^{i}(x)-x\right|=\left|a^{i}(y)-y\right|$. Consider all $i \geq 0$ with $\left|a^{i}(x)-x\right|=\left|a^{i}(y)-y\right| \leq R$. Then by the acylindricity, since the $a^{i}$ 's are all distinct from each other, there exists at least one $I$ with $0 \leq I \leq K(R)$ such that $\left|a^{I}(x)-x\right|>R$. Since $x \in \alpha$, by Lemma 2, $R-2(\delta+\Delta)<I \operatorname{tr}(a)$; therefore $(R-2(\delta+\Delta)) / K(R)<\operatorname{tr}(a)$. Choose an integer $P$ with $K(R) /(R-2(\delta+\Delta)) \leq P$. Then $1<\operatorname{tr}\left(a^{P}\right)$. Now take $R=2(\delta+\Delta)+1$.

Convention 13 (Subscript of a constant). To keep track of constants, we will number a constant by the number of the claim in which the constant first appears; for example, the constant $P$ in Lemma 12 will be $R_{12}$. We may omit the subscript if there is no confusion.

We state one key lemma on the action of a commutator

$$
[a, b]=\left[a^{-1} b^{-1} a b\right] .
$$

In the following claim, when we say $X<Y$ and $X<p$, we choose and fix parameters for $X, Y, p$. Remember that we always have $X<a(X)$ and $X^{\prime}<b\left(X^{\prime}\right)$.

Lemma 14. Let $\Gamma$ be a $\delta$-hyperbolic space and $a, b$ hyperbolic isometries, with quasi-axes $\alpha, \beta$. 
Let $X, Y \in \alpha \cap N_{\Lambda}(\beta)$ be the extremal points with $X<Y$ on $\alpha$. Take $X^{\prime}, Y^{\prime} \in \beta$ with $\left|X-X^{\prime}\right|,\left|Y-Y^{\prime}\right| \leq \Lambda$.

Let $p \in \alpha$ be a point with $X<p$ such that

(i) $|X-p|+\operatorname{tr}(a)+\operatorname{tr}(b)+6 \delta+7 \Delta+4 \Lambda \leq|X-Y|$.

(ii) $10 \delta+23 \Delta+4 \Lambda \leq|X-p|$.

If $X^{\prime}<Y^{\prime}$ on $\beta$, then

$$
|p-([b, a])(p)| \leq 28 \delta+30 \Delta+12 \Lambda .
$$

We remark that if $X^{\prime}>Y^{\prime}$, then the inequality holds for $\left[b^{-1}, a\right]$ instead of $[b, a]$. The argument is the same after replacing $b$ by $b^{-1}$.

Proof. Notice that the claim is trivial if $\delta=0$. If $\delta=0$, then $\Lambda=0$, and $[X, Y]=$ $\alpha \cap \beta$ can be viewed as an interval such that $a$ is a translation by $\operatorname{tr}(a)$, and $b$ is a translation by $\operatorname{tr}(b)$. Therefore $b^{-1} a^{-1} b a(p)=p+\operatorname{tr}(a)+\operatorname{tr}(b)-\operatorname{tr}(a)-\operatorname{tr}(b)=p$ if $|X-p|+\operatorname{tr}(a)+\operatorname{tr}(b) \leq|X-Y|$.

In general, we deal with the error terms. For this we need to verify, for example, that $p, a(p)<Y$. We will argue that later using (i) and (ii). The condition (i) is a replacement for $|X-p|+\operatorname{tr}(a)+\operatorname{tr}(b) \leq|X-Y|$, and (ii) says that $p$ is not so close to $X$.

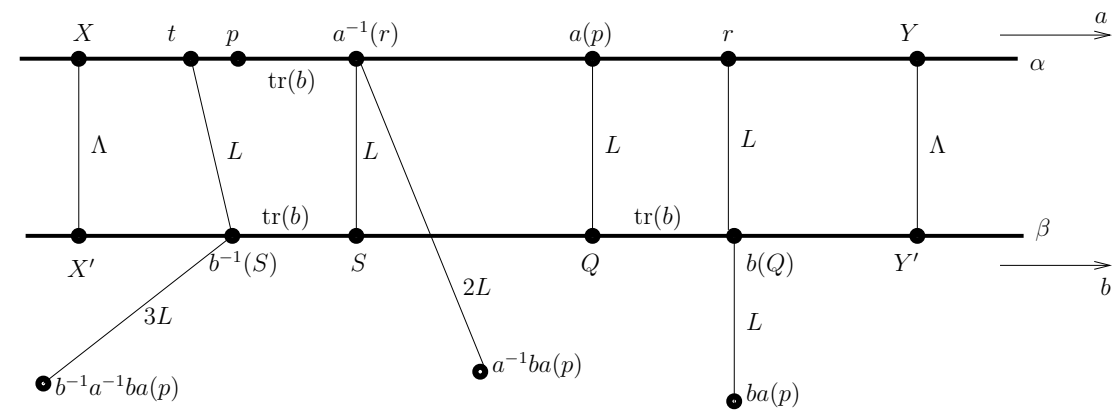

Figure 2. Action by $[b, a]$

Set $\alpha^{\prime}=\alpha_{X, Y}$, and $\beta^{\prime}=\beta_{X^{\prime}, Y^{\prime}}$. For any point $q \in \alpha^{\prime}$, there is a point $Q \in \beta^{\prime}$ with $\left|q-Q^{\prime}\right| \leq \Lambda+2 \delta+2 \Delta=L$, and vice versa.

Take $Q \in \bar{\beta}^{\prime}$ with $|a(p)-Q| \leq L$ (we will argue $a(p) \in \alpha^{\prime}$ ). Take $r \in \alpha^{\prime}$ with $|b(Q)-r| \leq L$ (we will argue $\left.b(Q) \in \beta^{\prime}\right)$. Then, $|b a(p)-r| \leq|b a(p)-b(Q)|+$ $|b(Q)+r| \leq 2 L$.

Take $S \in \beta^{\prime}$ with $\left|S-a^{-1}(r)\right| \leq L$ (we will argue $a^{-1}(r) \in \alpha^{\prime}$ ). Then, $\left|S-a^{-1} b a(p)\right| \leq\left|S-a^{-1}(r)\right|+\left|a^{-1}(r)-a^{-1} b a(p)\right| \leq L+2 L=3 L$.

Notice that $\left|a^{-1}(r)-p\right|$ is approximately $\operatorname{tr}(b)$; therefore $b^{-1}(S)$ is close to $p$. To be precise, let $t \in \alpha^{\prime}$ be with $\left|t-b^{-1}(S)\right| \leq L\left(b^{-1}(S) \in \beta^{\prime}\right.$ by (ii)). Then $\operatorname{tr}(b)-2 L \leq\left|t-a^{-1}(r)\right| \leq 2 L+\left|S-b^{-1}(S)\right| \leq \operatorname{tr}(b)+2(\delta+\Delta+L)$. Also, $\operatorname{tr}(b) \leq|Q-b(Q)| \leq \operatorname{tr}(b)+2(\delta+\Delta)$; therefore $\operatorname{tr}(b)-2 L \leq|a(p)-r|=\left|p-a^{-1}(r)\right| \leq$ $\operatorname{tr}(b)+2(\delta+\Delta+2 L)$.

Now, $t, p, a^{-1}(r)$ are on the quasi-axes $\alpha$. Let's assume that $p$ is between $t$ and $a^{-1}(r)$ (the same argument applies if $t$ is between $p$ and $a^{-1}(r)$ ). Then there is a point $p^{\prime} \in\left[t, a^{-1}(r)\right]$ with $\left|p-p^{\prime}\right| \leq \Delta$. It follows that $|t-p| \leq 4(\delta+\Delta+2 L)+2 \Delta$. 
We get $\left|p-b^{-1} a^{-1} b a(p)\right| \leq\left|b^{-1} a^{-1} b a(p)-b^{-1}(S)\right|+\left|b^{-1}(S)-t\right|+|t-p| \leq$ $3 L+L+4(\delta+\Delta+2 L)+2 \Delta=4 \delta+6 \Delta+12 L=28 \delta+30 \Delta+12 \Lambda$. The inequality is proved.

We are left to verify the claims on the positions of the points we used in the argument. First, we show $p<Y$ using (i). Since $|X-p| \leq|X-Y|-\Delta$ (by (i)), it follows that $p<Y$. Indeed, otherwise, i.e. $Y$ is between $X$ and $p$, there must be a point $y \in[X, p]$ with $|Y-y| \leq \Delta$, which gives a contradiction. Next, $X<a(p)<Y$. $X<a(X)$ is trivial, and $a(p)<Y$ is by an argument similar to the one for $p<Y$, so we omit it.

We will show that (i) implies that $b(Q)$ is between $X^{\prime}$ and $Y^{\prime}$; therefore $r$ exists. It suffices if we check that

(0) $\left|X^{\prime}-b(Q)\right| \leq\left|X^{\prime}-Y^{\prime}\right|-\Delta$.

To see that, first,

(1) $\left|X^{\prime}-Q\right| \leq|X-p|+|p-a(p)|+\Lambda+L \leq|X-p|+\operatorname{tr}(a)+2(\delta+\Delta)+\Lambda+L$.

Next, using (1),

(2) $\left|X^{\prime}-b(Q)\right| \leq\left|X^{\prime}-Q\right|+|Q-b(Q)| \leq|X-p|+\operatorname{tr}(a)+2(\delta+\Delta)+\Lambda+L+$ $\operatorname{tr}(b)+2(\delta+\Delta) \leq|X-p|+\operatorname{tr}(a)+\operatorname{tr}(b)+4(\delta+\Delta)+\Lambda+L$.

Also,

(3) $\left|X^{\prime}-Y^{\prime}\right| \geq|X-Y|-2 \Lambda$.

By (2) and (3), (0) follows if $|X-p|+\operatorname{tr}(a)+\operatorname{tr}(b)+4(\delta+\Delta)+\Lambda+L+2 \Lambda+\Delta \leq$ $|X-Y|$. But this is (i) since $4(\delta+\Delta)+\Lambda+L+2 \Lambda+\Delta=6 \delta+7 \Delta+4 \Lambda$.

That $a^{-1}(r)$ is between $X$ and $r$ is easy, so we omit a proof and show that (ii) implies that $b^{-1}(S)$ is between $X^{\prime}$ and $Y^{\prime}$. It suffices if

(4) $\left|Y^{\prime}-b^{-1}(S)\right| \leq\left|Y^{\prime}-X^{\prime}\right|-\Delta$.

First,

(5) $\left|X^{\prime}-Q\right| \geq|X-a(p)|-L-\Lambda$.

Next, using (5) (we use that $b^{-1}$ maps $S$ toward $X^{\prime}$ for the first inequality),

(6) $\left|X^{\prime}-Y^{\prime}\right| \geq\left|X^{\prime}-Q\right|+|Q-b(Q)|+\left|b(Q)-Y^{\prime}\right|-4 \Delta \geq(|X-a(p)|-\Lambda-L)+$ $(\operatorname{tr}(b)-2 \Delta)+\left|b(Q)-Y^{\prime}\right|-4 \Delta \geq(|X-p|+\operatorname{tr}(a)-2 \Delta-\Lambda-L)+(\operatorname{tr}(b)-2 \Delta)+$ $\left|b(Q)-Y^{\prime}\right|-4 \Delta=|X-p|+\left|b(Q)-Y^{\prime}\right|+\operatorname{tr}(a)+\operatorname{tr}(b)-8 \Delta-\Lambda-L$.

Also,

(7) $\left|Y^{\prime}-b^{-1}(S)\right| \leq\left|Y^{\prime}-b(Q)\right|+|b(Q)-S|+\left|S-b^{-1}(S)\right|+4 \Delta \leq\left|Y^{\prime}-b(Q)\right|+$ $(\operatorname{tr}(a)+2(\delta+\Delta)+2 L)+(\operatorname{tr}(b)+2(\delta+\Delta))+4 \Delta=\left|Y^{\prime}-b(Q)\right|+\operatorname{tr}(a)+\operatorname{tr}(b)+$ $4 \delta+8 \Delta+2 L$.

Using (6) and (7), (4) follows from $4 \delta+17 \Delta+\Lambda+3 L \leq|X-p|$. But this is the same as (ii) since $4 \delta+17 \Delta+\Lambda+3 L=10 \delta+23 \Delta+4 \Lambda$.

Lemma 15. Let $\Gamma$ be a $\delta$-hyperbolic space and $G$ a group acting on $\Gamma$ acylindrically with constants $K(R), L(R)$. Suppose $a, b \in G$ act hyperbolically with quasi-axes $\alpha, \beta \subset \Gamma$, respectively.

If $a^{n} b \neq b a^{n}$ for all $n \neq 0$ or $b^{n} a \neq a b^{n}$ for all $n \neq 0$, then

$$
\left|\alpha \cap_{\Lambda} \beta\right|<L+(1+K) \max (\operatorname{tr}(a), \operatorname{tr}(b))+16 \delta+36 \Delta+10 \Lambda,
$$

where $K=K(28 \delta+30 \Delta+12 \Lambda), L=L(28 \delta+30 \Delta+12 \Lambda)$.

Proof. To argue by contradiction, assume that the inequality was false. Let $X, Y \in$ $\alpha \cap N_{\Lambda}(\beta)$ be the extremal points. Then, since $\left|\alpha \cap_{\Lambda} \beta\right|-2 \Delta-2 \Lambda \leq|X-Y|$, we have $|X-Y| \geq L+(1+K) \max (\operatorname{tr}(a), \operatorname{tr}(b))+16 \delta+34 \Delta+8 \Lambda$. 
Then we can take $p, q \in \alpha$ between $X$ and $Y$ such that

$$
10 \delta+23 \Delta+4 \Lambda \leq|X-p| \leq|X-q| \leq|X-Y|-\operatorname{tr}(a)-K \operatorname{tr}(b)+6 \delta+7 \Delta+4 \Lambda
$$

and $|p-q| \geq L$. Indeed, choose $P, Q \in[X, Y]$ with $|X-P|=10 \delta+23 \Delta+4 \Lambda+\Delta$ and $|X-Q|=|X-P|+L+2 \Delta$. Then $|Q-Y| \geq(1+K) \max (\operatorname{tr}(a), \operatorname{tr}(b))+6 \delta+$ $7 \Delta+4 \Lambda+\Delta$. Now take $p, q \in \alpha$ with $|P-p| \leq \Delta,|Q-q| \leq \Delta$.

Now, for each $n, 0 \leq n \leq K$, the conditions (i) and (ii) in Lemma 14 are satisfied by $a$ and $b^{n}$ (or $a$ and $b^{-n}$. See the remark after the lemma. Then we use $b^{-n}$. This is enough for our purpose) with respect to $p$ and also $q$. Therefore, by the lemma, each $g=\left[a, b^{n}\right], 0 \leq n \leq K$ satisfies $|p-g(p)|,|q-g(q)| \leq 28 \delta+30 \Delta+12 \Lambda$.

On the other hand, by the acylindricity, there are at most $K$ such elements $g$, so that there must be $0 \leq I<J \leq K$ such that $\left[a, b^{I}\right]=\left[a, b^{J}\right]$. This implies that $a b^{I-J}=b^{I-J} a$, a contradiction.

Similarly, by looking at the action by $\left[b, a^{n}\right], 0 \leq n \leq K$, we obtain $b a^{I-J}=$ $a^{I-J} b$ for some $0 \leq I<J \leq K$.

We state another lemma under the extra assumption that $\operatorname{tr}(a)=\operatorname{tr}(b)$, which we use later.

Lemma 16. Let $\Gamma$ be a $\delta$-hyperbolic space, and $G$ a group acting on $\Gamma$ acylindrically with constants $K(R), L(R)$. Suppose $a, b \in G$ act hyperbolically with quasi-axes $\alpha, \beta$. Let $X, Y \in \alpha$ be the extremal points of $\alpha \cap N_{\Lambda}(\beta)$ with $X<Y$, and $X^{\prime}, Y^{\prime} \in \beta$ with $\left|X-X^{\prime}\right| \leq \Lambda,\left|Y-Y^{\prime}\right| \leq \Lambda$. Assume $\operatorname{tr}(a)=\operatorname{tr}(b)$.

(I) Assume $X^{\prime}<b\left(X^{\prime}\right)$ on $\beta$. Let $p \in \alpha$ be a point between $X$ and $Y$ such that

$$
6 \delta+6 \Delta+2 \Lambda \leq|X-p| \leq|X-Y|-\operatorname{tr}(a)-2 \delta-3 \Delta ;
$$

then

$$
\left|b^{-1} a(p)-p\right| \leq 8 \delta+9 \Delta+2 \Lambda
$$

(II) If $a^{n} \neq b^{n}$ and $a^{n} \neq b^{-n}$ for all $n>0$, then

$$
\left|\alpha \cap_{\Lambda} \beta\right|<L+(1+K) \operatorname{tr}(a)+7 \delta+13 \Delta+2 \Lambda,
$$

where $K=K(8 \delta+9 \Delta+2 \Lambda), L=L(8 \delta+9 \Delta+2 \Lambda)$.

Proof. We omit details since it is similar to (and less complicated than) Lemma 15 and Lemma 14. The outline for (I) is that with the extra assumption $\operatorname{tr}(a)=\operatorname{tr}(b)$, a point $p \in \alpha \cap_{\Lambda} \beta$ does not move very much by $b^{-1} a$. Notice that if $\Gamma$ is a tree, then $p$ does not move. As in Lemma 14 we obtain the upper bound on $\left|p-b^{-1} a(p)\right|$. Remember that we always have $X<a(X)$. For (II), by considering $b^{-1}$ instead of $b$ if necessary, we may assume $X^{\prime}<b\left(X^{\prime}\right)$ (after possibly replacing $b$ by $b^{-1}$ ). Then, as in Lemma 15, it follows from (I) and the acylindricity that $b^{-n} a^{n}=b^{-m} a^{m}$ for some $n<m$; therefore, $a^{m-n}=b^{m-n}$, a contradiction.

Combining Proposition 8 and Lemma 15, we obtain the following. This says that we can find a global bound on one of the exponents, but the other bound depends on the ratio $\operatorname{tr}(a) / \operatorname{tr}(b)$.

Proposition 17. Let $G$ be a group which acts on a $\delta$-hyperbolic space $\Gamma$ acylindrically with constants $K(R), L(R)$. Then, there exists a constant $N=N_{17} \geq 1$ with the following property. $N$ depends only on $\delta, K(28 \delta+30 \Delta+12 \Lambda), L(28 \delta+30 \Delta+12 \Lambda)$ and $K(2(\delta+\Delta)+1)$. 
Suppose $a, b \in G$ act hyperbolically. Assume $\left[a^{n}, b^{m}\right] \neq 1$ for all $n, m \neq 0$. Suppose there exists a number $q \geq 1$ such that

$$
\operatorname{tr}(a) / q \leq \operatorname{tr}(b) \leq \operatorname{tr}(a) .
$$

Then, for any $n \geq N$ and $m \geq q N,\left\langle a^{n}, b^{m}\right\rangle$ is a free group freely generated by $a^{n}, b^{m}$. Moreover, the embedding of $\left\langle a^{n}, b^{m}\right\rangle$ by an orbit in $\Gamma$ is quasi-isometric.

Proof. Put $K=K(28 \delta+30 \Delta+12 \Lambda), L=L(28 \delta+30 \Delta+12 \Lambda)$. It then suffices to set

$$
N=(1+K)+P_{12}(42 \delta+50 \Delta+14 \Lambda+L+2) .
$$

By Lemma 15] since $\operatorname{tr}(b) \leq \operatorname{tr}(a) \leq q \operatorname{tr}(b)$, we have $\left|\alpha \cap_{\Lambda} \beta\right|<L+(1+K) \operatorname{tr}(a)+$ $16 \delta+36 \Delta+10 \Lambda$ and $\left|\alpha \cap_{\Lambda} \beta\right|<L+(1+K) q \operatorname{tr}(b)+16 \delta+36 \Delta+10 \Lambda$. Therefore, if $n \geq N$ and $m \geq q N$, then both $\operatorname{tr}\left(a^{n}\right)$ and $\operatorname{tr}\left(b^{m}\right)$ are $\geq(1+K) \operatorname{tr}(a)+42 \delta+$ $50 \Delta+14 \Lambda+L+2>\left|\alpha \cap_{\Lambda} \beta\right|+26 \delta+14 \Delta+4 \Lambda+2$. Therefore the conditions of Proposition 8 are satisfied by $a^{n}, b^{m}$ with the constant $U=1$, so that $\left\langle a^{n}, b^{m}\right\rangle$ is free and the embedding is quasi-isometric.

3.4. Another condition for freeness. In this section we discuss the case when $\operatorname{tr}(b) \ll \operatorname{tr}(a)$; otherwise we can apply Proposition 17 with the constant $q$ controlled.

Proposition 18. Suppose $G$ acts on a $\delta$-hyperbolic space $\Gamma$ acylindrically with constants $K(R), L(R)$. Then there exists a constant $N=N[18>0$ with the following property. $N$ depends only on $\delta, K(8 \delta+9 \Delta+2 \Lambda), L(8 \delta+9 \Delta+2 \Lambda)$.

Assume that $f, g \in G$ act hyperbolically with quasi-axes $\alpha, \beta$ such that $\left[f^{s}, g^{t}\right] \neq 1$ for any $s, t \neq 0$. Suppose that the following conditions are satisfied:

(1) $\operatorname{tr}(g) \leq \operatorname{tr}(f)$

(2) $\left|\alpha \cap_{\Lambda} \beta\right| \leq 2 \operatorname{tr}(f)$

If $n \geq N$, then $\left\langle g, f^{n}\right\rangle$ is a free group freely generated by $g, f^{n}$. Moreover, the embedding of $\left\langle g, f^{n}\right\rangle$ in $\Gamma$ by an orbit is quasi-isometric.

Proof. $\left|\alpha \cap_{\Lambda} \beta\right|<\infty$ by our assumption. As in the proof for Proposition 8 , we only discuss the case $\alpha \cap_{\Lambda} \beta \neq \emptyset$ in detail.

Suppose $\alpha \cap_{\Lambda} \beta \neq \emptyset$. Let $S, T \in \alpha$ be the extremal points for $\alpha \cap_{\Lambda} \beta$. Let $Q$ be the mid-point of $[S, T]=\ell$, and take $m \in \alpha$ with $|m-Q| \leq \Delta$. Then $m \in N_{2 \delta+2 \Delta+\Lambda}(\beta)$.

Set $K=K(8 \delta+9 \Delta+2 \Lambda), L=L(8 \delta+9 \Delta+2 \Lambda)$ (see Lemma 16 (II)), and

$$
N=\left(17+2 K+\frac{1+2 L+4 Z}{P}\right) \text {, }
$$

where $Z$ is a constant we decide later, which depends only on $\delta, \Delta, \Lambda$. It follows that if $n \geq N$, then $\operatorname{tr}\left(f^{n}\right) \geq(17+2 K) \operatorname{tr}(f)+1+2 L+4 Z$. We will show this $N$ is a desired constant if we choose $Z$ appropriately. To use it later, we choose $Q$ such that

$$
\operatorname{tr}\left(f^{4}\right)+Z \leq \operatorname{tr}\left(g^{Q}\right) \leq \operatorname{tr}\left(f^{5}\right)+Z .
$$

This is possible because of the assumption $\operatorname{tr}(g) \leq \operatorname{tr}(f)$. This is the only place where we use this assumption. Then, by the second inequality in (3),

$$
\operatorname{tr}\left(f^{n}\right) \geq 3 \operatorname{tr}\left(g^{Q}\right)+2 L+2(1+K) \operatorname{tr}(f)+Z .
$$

Also, since $2 \operatorname{tr}(f) \geq\left|\alpha \cap_{\Lambda} \beta\right|$, by the first inequality in (3),

$$
\operatorname{tr}\left(g^{Q}\right) \geq 2\left|\alpha \cap_{\Lambda} \beta\right|+Z \text {. }
$$


For $n \geq N$, set $a=f^{n}, b=g$. Now set

$$
Z=1+40 \delta+32 \Delta+6 \Lambda \text {. }
$$

It is a good idea to have the following rough estimates in our mind:

$$
1+\delta+\Delta+\Lambda+\left|\alpha \cap_{\Lambda} \beta\right| \ll \operatorname{tr}\left(b^{Q}\right) \ll \operatorname{tr}(a) .
$$

By Lemma 2, we have $|m-a(m)| \geq\left|m-b^{Q}(m)\right|$.

We start the argument. We emphasize that the argument will only rely on (4), (5) and the definition of $Z$. Let $\Omega(a, b)$ be the Cayley graph of the free group freely generated by $a, b$. Here we abused $a, b$ to define the free group $F(a, b)$ abstractly.

We will show that there exists a constant $V>0$ such that for all non-empty reduced words $w$ on $a, b$,

$$
V|w| \leq|w(m)-m|
$$

Since our embedding is by the action, it follows that for any reduced words $v, w$ which are elements in the free group $F(a, b)$, we have $V|v-w| \leq|v(m)-w(m)|$, where $|v-w|$ is the distance between $v$ and $w$ on $F(a, b)$. The inequality says that $a, b$ freely generate a free group of rank two in $\operatorname{Isom}(\Gamma)$.

The idea of the proof is the same as for Proposition 8 but we choose points in $\Gamma$ between $m$ and $w(m)$ more carefully and verify that they satisfy the three points condition for $U=1$.

There are three cases according to the form of $w$ :

(0) $w=b^{m}(m \neq 0)$,

(1) $w=a^{n_{1}} b^{m_{1}} \cdots a^{n_{i}} b^{m_{i}}(i \geq 1)$ such that $n_{1} \neq 0, n_{i} \neq 0$ and $m_{i}$ is possibly 0 ,

(2) $w=b^{m_{0}} a^{n_{1}} b^{m_{1}} \cdots a^{n_{i}} b^{m_{i}}(i \geq 1)$ such that $m_{0} \neq 0, n_{i} \neq 0$ and $m_{i}$ is possibly 0 .

It is enough if we find a desired constant $V$ for each case (then take the minimum of those three numbers). Since $b$ is hyperbolic, by definition, such $V>0$ exists for $(0)$. For (1) and (2), the argument is similar and we will only discuss (1) in detail.

In the tree $\Omega$, on the geodesic from 1 to $w$, there are the following vertices, as many as $|w|+1$, in this order (again, we assume $n_{j}, m_{j}>0$ for simplicity):

$$
\begin{aligned}
& 1, a, a^{2}, \cdots, a^{n_{1}} \\
& a^{n_{1}} b, \cdots, a^{n_{1}} b^{m_{1}} \\
& a^{n_{1}} b^{m_{1}} a, \cdots, a^{n_{1}} b^{m_{1}} a^{n_{2}} \\
& \cdots \\
& \quad a^{n_{1}} b^{m_{1}} \cdots a^{n_{i}} b, \cdots, a^{n_{1}} b^{m_{1}} \cdots a^{n_{i}} b^{m_{i}}=w .
\end{aligned}
$$

If we apply those elements to the point $m$, we obtain a sequence of points in $\Gamma$. In general, this sequence does not satisfy the three points condition, but we will choose a subsequence which will satisfy the condition.

To define points, we write (uniquely) for each $j$,

$$
m_{j}=o_{j} Q+l_{j},
$$

such that $o_{j} \in \mathbb{Z}$ and $0 \leq l_{j}<Q$ if $m_{j} \geq 0$ and $-Q<l_{j} \leq 0$ if $m_{j}<0$. 
Now define a sequence, which is a subsequence of the previous one, as follows:

$$
\begin{aligned}
& 1, a, a^{2}, \cdots, a^{n_{1}} \\
& a^{n_{1}} b^{Q}, a^{n_{1}} b^{2 Q}, \cdots, a^{n_{1}} b^{\left(o_{1}-1\right) Q}, a^{n_{1}} b^{m_{1}} \\
& a^{n_{1}} b^{m_{1}} a, a^{n_{1}} b^{m_{1}} a^{2}, \cdots, a^{n_{1}} b^{m_{1}} a^{n_{2}} \\
& a^{n_{1}} b^{m_{1}} a^{n_{2}} b^{Q}, \cdots, a^{n_{1}} b^{m_{1}} a^{n_{2}} b^{\left(o_{2}-1\right) Q}, a^{n_{1}} b^{m_{1}} a^{n_{2}} b^{m_{2}} \\
& \quad \cdots \\
& a^{n_{1}} b^{m_{1}} \cdots a^{n_{i}} b^{Q}, \cdots, a^{n_{1}} b^{m_{1}} \cdots a^{n_{i}} b^{m_{i}}=w .
\end{aligned}
$$

The odd rows are the same as before, but for each even row, we remove points except for every $Q$-th one and the last one (instead we remove $a^{n_{1}} b^{o_{1} Q}$, for example, in the second row). Therefore, the $2 j$-th row will be empty if $\left|m_{j}\right|<Q$. But for the $2 i$-th row (the last one), even if $\left|m_{i}\right|<Q$, we do not remove $w$. Clearly, this sequence contains at least $1+[|w| / Q]$ points, where $[x]$ is the largest integer which is not larger than $x$. We will show that the orbit of the point $m$ by this sequence of elements satisfies the three points condition for $U=1$. It then follows from Proposition 7 that $(1+[|w| / Q]) \leq|w(m)-m|$. We are done since $|w| / Q \leq(1+[|w| / Q])$ by setting $V=1 / Q$. What is important is that although the constant $Q$ may depend on $a$ and $b$, it does not depend on the word $w$.

The rest of the argument is to verify the three points condition. Namely, for $A, B, C$, we want (the constant is 1 )

$$
1+2 \delta \leq \min (|A-B|,|B-C|)-(|A-B|+|B-C|-|A-C|) .
$$

In this proof, we write the right hand side of the inequality as $\operatorname{RHS}(A, B, C)$ or RHS. Using the Gromov product, $|A-B|+|B-C|-|A-C|=2(A, C)_{B}$, which is $\leq 2\left([A, C] \cap_{\delta}[B, C]\right)+6 \delta$ by Lemma 6 .

We only discuss the case such that the three points are contained in the first three rows (the same argument applies to the other cases). Regarding the positions of the three points, there are cases, which slightly differ depending on $o_{1}=0,1$ or $>1$. As usual we assume $n_{1}, m_{1}, n_{2}>0$ to simplify the notation.

$o_{1}>1$ :

(I) $a^{k-1}(m), a^{k}(m), a^{k+1}(m)$.

(II) $a^{n_{1}-1}(m), a^{n_{1}}(m), a^{n_{1}} b^{Q}(m)$.

(III) $a^{n_{1}} b^{(k-1) Q}(m), a^{n_{1}} b^{k Q}(m), a^{n_{1}} b^{(k+1) Q}(m)$.

(IV) $a^{n_{1}} b^{\left(o_{1}-2\right) Q}(m), a^{n_{1}} b^{\left(o_{1}-1\right) Q}(m), a^{n_{1}} b^{m_{1}}(m)$.

(V) $a^{n_{1}} b^{\left(o_{1}-1\right) Q}(m), a^{n_{1}} b^{m_{1}}(m), a^{n_{1}} b^{m_{1}} a(m)$.

(VI) $a^{n_{1}} b^{m_{1}} a^{k-1}(m), a^{n_{1}} b^{m_{1}} a^{k}(m), a^{n_{1}} b^{m_{1}} a^{k+1}(m)$.

$o_{1}=1$ :

(II)-(V) do not occur. Instead there are extra cases:

(II-I) $a^{n_{1}-1}(m), a^{n_{1}}(m), a^{n_{1}} b^{m_{1}}(m)$.

$(\mathrm{V}-\mathrm{I}) a^{n_{1}}(m), a^{n_{1}} b^{m_{1}}(m), a^{n_{1}} b^{m_{1}} a(m)$.

$o_{1}=0$ :

(II)-(V) do not occur, but there are extra cases:

(II-II): $a^{n_{1}-1}(m), a^{n_{1}}(m), a^{n_{1}} b^{m_{1}} a(m)$.

(V-II): $a^{n_{1}}(m), a^{n_{1}} b^{m_{1}} a(m), a^{n_{1}} b^{m_{1}} a^{2}(m)$.

Before we discuss each case, we treat the essential case in advance. 
Lemma 19. The three points condition is satisfied with constant $U=1$ for $\left(a^{ \pm 1}(m)\right.$, $\left.m, b^{ \pm Q}(m)\right)$.

Proof. We only prove it for $\left(a(m), m, b^{Q}(m)\right)$. The other cases are the same. First, we have $|m-a(m)| \geq\left|m-b^{Q}(m)\right| \geq \operatorname{tr}\left(b^{Q}\right)-2(2 \delta+2 \Delta+\Lambda)$. So,

$$
\begin{aligned}
\operatorname{RHS} & \left(a(m), m, b^{Q}(m)\right) \\
& \geq\left(\operatorname{tr}\left(b^{Q}\right)-2(2 \delta+2 \Delta+\Lambda)\right)-2\left|[a(m), m] \cap_{\delta}\left[m, b^{Q}(m)\right]\right|-6 \delta \\
& \geq\left(\operatorname{tr}\left(b^{Q}\right)-2(2 \delta+2 \Delta+\Lambda)\right)-2\left(\left|\alpha \cap_{\Lambda} \beta\right|+2(2 \delta+2 \Delta+\Lambda)+10 \delta\right)-6 \delta \\
& \geq Z-2(2 \delta+2 \Delta+\Lambda)-2(2(2 \delta+2 \Delta+\Lambda)+10 \delta)-6 \delta \\
& =Z-38 \delta-12 \Delta-6 \Lambda \geq(1+2 \delta)
\end{aligned}
$$

by (5). $Z$ was chosen to satisfy this (and one more requirement later).

We discuss each case. The case with $o_{1}>1$ is straightforward.

(I) Those three points are on the quasi-axis $\alpha$ for $a$ in this order. By Lemma 2. RHS $\geq \operatorname{tr}(a)-4(\delta+\Delta) \geq Z-4(\delta+\Delta) \geq(1+2 \delta)$ by (4).

(II) Apply $a^{-n_{1}}$ and get $a^{-1}(m), m, b^{Q}(m)$. The three points conditions is already known by Lemma 19 .

(III) Similar to (I). Apply $a^{-n_{1}}$; then the three points $b^{(k-1) Q}(m), b^{k Q}(m)$, $b^{(k+1) Q}(m)$ are in $N_{2 \delta+2 \Delta+\Lambda}(\beta)$. It follows that RHS $\geq \operatorname{tr}\left(b^{Q}\right)-4(\delta+\Delta)-$ $4(2 \delta+2 \Delta+\Lambda) \geq Z-12 \delta-12 \Delta-4 \Lambda \geq(1+2 \delta)$ by (5).

(IV) Same as (III). Apply $b^{\left(1-o_{1}\right) Q} a^{-n_{1}}$; then we get $b^{-Q}(m), m, b^{m_{1}}(m)$. Use $m_{1} \geq Q$. Then, RHS $\geq \operatorname{tr}\left(b^{Q}\right)-6(\delta+\Delta)-4(2 \delta+2 \Delta+\Lambda) \geq(1+2 \delta)$ by (5), in particular $\operatorname{tr}\left(b^{Q}\right) \geq Z$.

(V) Similar to (II). Apply $b^{-m_{1}} a^{-n_{1}}$; then we get $b^{\left(o_{1}-1-m_{1}\right) Q}(m), m, a(m)$. First $\left|m-b^{\left(o_{1}-1-m_{1}\right) Q}(m)\right| \leq|m-a(m)|$. Also, $o_{1}-1-m_{1}<-1$. The rest is the same as (II) (namely, we show something similar to Lemma 19), and we omit it.

(VI) Exactly the same as (I). Apply $a^{-k} b^{-m_{1}} a^{-n_{1}}$; then get $a^{-1}(m), m, a(m)$.

The case with $o_{1}=1$ is similar to the case with $o_{1}>1$. Note that $2 Q>m_{1} \geq Q$.

(II-I) Similar to (II). Apply $a^{-n_{1}}$ and get $a^{-1}(m), m, b^{m_{1}}(m)$. Use $m_{1} \geq Q$. First $\left|m-a^{-1}(m)\right| \geq\left|m-b^{m_{1}}(m)\right|$. The rest is the same and we omit it.

(V-I) Apply $b^{-m_{1}} a^{-n_{1}}$; then we get $b^{-m_{1}}(m), m, a(m)$. The same estimate as (II-I) holds.

The case for $o_{1}=0$, i.e., $0 \leq m_{1}<Q$, is slightly more complicated.

(II-II) Apply $a^{-n_{1}}$ and get $a^{-1}(m), m, b^{m_{1}} a(m)$. To show the three points condition for $U=1$ for this, we insert one point $b^{m_{1}}(m)$. (This point corresponds to a point we omitted when we defined the subsequence.) We now have

$$
a^{-1}(m), m, b^{m_{1}}(m), b^{m_{1}} a(m) .
$$

We state a lemma from elementary $\delta$-hyperbolic geometry. We leave the proof to the reader (draw a geodesic $[C, B]$ and argue). We remark that in a tree, the equality holds without the constant.

Lemma 20. Let $A, B, C, D$ be four points in a $\delta$-hyperbolic space. Then,

$$
|A-C| \geq|A-B|+|C-D|-|B-D|-2\left|[A, B] \cap_{3 \delta}[C, D]\right|-10 \delta .
$$


It follows from the lemma that $|A-B|+|B-C|-|A-C| \leq|A-B|+|B-C|-$ $|A-B|-|C-D|+|B-D|+2\left|[A, B] \cap_{3 \delta}[C, D]\right|+10 \delta \leq 2|[A, B] \cap 3 \delta[C, D]|+2|B-D|$ $+10 \delta$.

Therefore, $\operatorname{RHS}(A, B, C)=\min (|A-B|,|B-C|)-(|A-B|+|B-C|-|A-C|) \geq$ $\min (|A-B|,|C-D|)-|B-D|-\left(2\left|[A, B] \cap_{3 \delta}[C, D]\right|+2|B-D|+10 \delta\right)$.

Now, let $A=a^{-1}(m), B=m, D=b^{m_{1}}(m), C=b^{m_{1}} a(m)$ (see Figure 3). Then by $|A-B|=|C-D|$ and Lemma 2, the last term in the above estimate is $\geq$ $\operatorname{tr}(a)-3 \operatorname{tr}\left(b^{m_{1}}\right)-6(\delta+\Delta)-10 \delta-2\left|[A, B] \cap_{3 \delta}[C, D]\right| \geq 2 L+2(1+K) \operatorname{tr}(f)+Z-$ $16 \delta-6 \Delta-2\left|[A, B] \cap_{3 \delta}[C, D]\right|$. The last inequality is by (44). We claim that this last term, $M$, is $\geq(1+2 \delta)$. For that, notice that $\left|[A, B] \cap_{3 \delta}[C, D]\right| \leq\left|\alpha \cap_{\Lambda} b^{m_{1}}(\alpha)\right|$, which is $\leq L+(1+K) \operatorname{tr}(f)+7 \delta+13 \Delta+2 \Lambda$ by the next lemma. Therefore, $M \geq Z-16 \delta-6 \Delta-2(7 \delta+13 \Delta+2 \Lambda)=Z-30 \delta-32 \Delta-4 \Lambda \geq(1+2 \delta)$. This is the other requirement we chose $Z$ to satisfy.

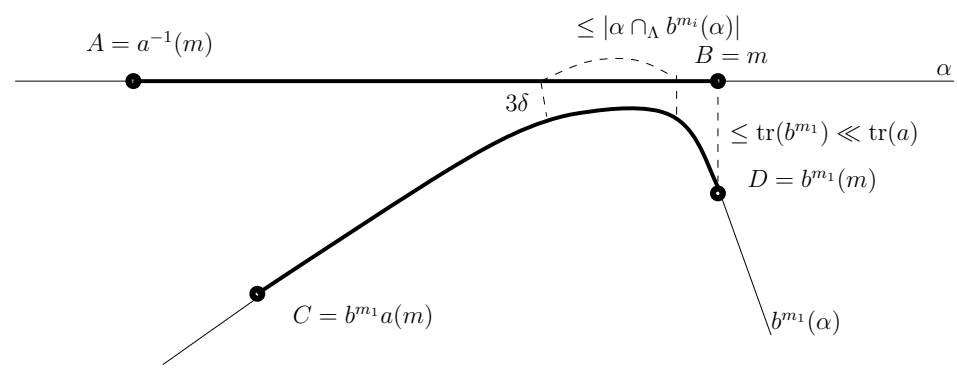

Figure 3. Case (II-II)

Lemma 21. For each $i \neq 0$,

$$
\left|\alpha \cap_{\Lambda} b^{i}(\alpha)\right| \leq L+(1+K) \operatorname{tr}(f)+7 \delta+13 \Delta+2 \Lambda .
$$

Proof. Since $\alpha$ is a quasi-axis for $f, b^{i}(\alpha)$ is a quasi-axis for $b^{i} f b^{-i}$. For any $N>0$, $f^{N} \neq\left(b^{i} f b^{-i}\right)^{N}$ and $f^{N} \neq\left(b^{i} f b^{-i}\right)^{-N}$ since otherwise $b^{i}$ and $f^{N}$ or $f^{-N}$ commute, which is impossible. Since $\operatorname{tr}(f)=\operatorname{tr}\left(b^{i} f b^{-i}\right)$, by Lemma 16 (II), we obtain the desired inequality.

(V-II) Apply $a^{-1} b^{-m_{1}} a^{-n_{1}}$ and get $a^{-1} b^{-m_{1}}(m), m, a(m)$. To estimate the RHS, insert a point $a^{-1}(m)$ and consider

$$
a^{-1} b^{-m_{1}}(m), a^{-1}(m), m, a(m) .
$$

We have $\left|a^{-1} b^{-m_{1}}(m)-a^{-1}(m)\right|=\left|b^{-m_{1}}(m)-m\right|$, and by this and the triangle inequality,

$\left|m-a^{-1}(m)\right|-\left|b^{-m_{1}}(m)-m\right| \leq\left|a^{-1} b^{-m_{1}}(m)-m\right| \leq\left|m-a^{-1}(m)\right|+\left|b^{-m_{1}}(m)-m\right|$.

Since $0 \leq m_{1}<Q$, roughly speaking, $\left|a^{-1} b^{-m_{1}}(m)-m\right|$ is comparable to $\left|m-a^{-1}(m)\right|$.

Now, RHS $\geq\left(|m-a(m)|-\left|m-b^{m_{1}}(m)\right|\right)-|m-a(m)|-(|m-a(m)|+$ $\left.\left|m-b^{m_{1}}(m)\right|\right)+\left(\left|a^{-1}(m)-a(m)\right|-\left|m-b^{m_{1}}(m)\right|\right)=\left|a^{-1}(m)-a(m)\right|-|m-a(m)|$ $-3\left|m-b^{m_{1}}(m)\right| \geq|m-a(m)|-2 \Delta-3\left|m-b^{m_{1}}(m)\right| \geq \operatorname{tr}(a)-2 \Delta-3 \operatorname{tr}\left(b^{m_{1}}\right)$ $-6(\delta+\Delta) \geq Z-6 \delta-8 \Delta \geq(1+2 \delta)$ by (4). We used $0 \leq m_{1}<Q$ (see Figure 4). 


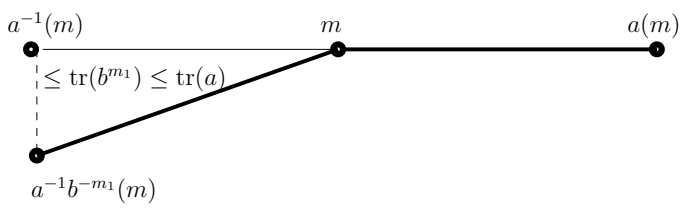

Figure 4. Case (V-II)

We completed the case (1). The case (2) is similar, and we omit the details. We finished the argument under the assumption that $\alpha \cap_{\Lambda} \beta \neq \emptyset$.

If $\alpha \cap_{\Lambda} \beta=\emptyset$, the argument is similar to the case with $\left|\alpha \cap_{\Lambda} \beta\right|>0$. Take the constants $K, L, P, Z, N$ as before. Set $a, b$ as before. Now let $\ell$ be a geodesic which realizes the distance between $\alpha, \beta$. Let $m$ be the mid-point of $\ell$. Then argue as before. We omit the details.

Before we state the main theorem, we state a proposition which can be shown similarly to Proposition 18. The conclusion is weaker since $N_{22}$ depends on $f, g$, but we do not require the conditions 1 and 2 in Proposition 18 regarding $\operatorname{tr}(f), \operatorname{tr}(g)$.

Proposition 22. Suppose $G$ acts on a $\delta$-hyperbolic space $\Gamma$ acylindrically with constants $K(R), L(R)$. Assume that $f, g \in G$ act hyperbolically with quasi-axes $\alpha, \beta$. Assume that $\left[f^{s}, g^{t}\right] \neq 1$ for any $s, t \neq 0$.

Then there exists a constant $N_{22]}=N>0$, which depends on $f, g$, such that if $n \geq N$, then $\left\langle g, f^{n}\right\rangle$ is a free group freely generated by $g, f^{n}$. Moreover, the embedding of $\left\langle g, f^{n}\right\rangle$ in $\Gamma$ by an orbit is quasi-isometric.

Proof. Most of the argument is identical to the proof of Proposition 18 and easier, since the difficult part was to obtain a uniform constant $N_{18}$ for all $f, g$.

We review the argument for Proposition 18 we first choose constants $K, L, Z$. Choose $Q$ which satisfies (5). Choose $N$ such that if $n \geq N$, then we have (4). We only need (4) and (5) for the rest of the argument.

In the current setting, since $\left|\alpha \cap_{\Lambda} \beta\right|<\infty$ by Lemma 15, we can choose $Q$ to satisfy (5). Then, we choose $N$ to have (4) if $n \geq N$. The rest of the argument is the same and we do not repeat.

3.5. Upper bound on both exponents. The following is the main theorem of Section 3

Theorem 23. Suppose $G$ acts acylindrically for constants $K(R), L(R)$ on a $\delta$ hyperbolic space $\Gamma$. Then there exists a constant $M_{23}$ with the following property, which depends only on $\delta$ and $K(R), L(R)$ for $R=2(\delta+\Delta)+1, R=8 \delta+9 \Delta+2 \Lambda$ and $R=28 \delta+30 \Delta+12 \Lambda$.

Suppose $a, b \in G$ act hyperbolically. Assume for any $p, q \neq 0,\left[a^{p}, b^{q}\right] \neq 1$ in $G$. Then for any $n, m \geq M,\left\langle a^{n}, b^{m}\right\rangle$ is a free group freely generated by $a^{n}, b^{m}$. Moreover, the embedding of $\left\langle a^{n}, b^{m}\right\rangle$ by an orbit in $\Gamma$ is quasi-isometric. In particular, all non-trivial elements in $\left\langle a^{n}, b^{m}\right\rangle$ are hyperbolic on $\Gamma$.

As for the dependence of $M$ on $K(R), L(R)$, only $R=2(\delta+\Delta)+1,8 \delta+9 \Delta+2 \Lambda$, and $28 \delta+30 \Delta+12 \Lambda$ matter. Recall that $\Delta=418 \delta, \Lambda=2 \Delta+3 \delta$.

Proof. Set $K=K(28 \delta+30 \Delta+12 \Lambda), L=L(28 \delta+30 \Delta+12 \Lambda)$ (from Lemma 15). Take constants $P_{12}, N_{17}$ and $N_{18}$ by Lemma 12, Proposition [17 and Proposition 
18. Those constants depend on $K(R), L(R)$ with the specific values of $R$. Set

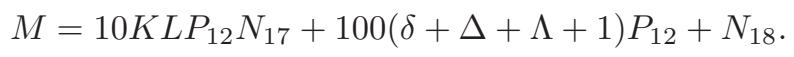

We will show

Claim. If $n, m \geq M,\left\langle a^{n}, b^{m}\right\rangle$ is a free group freely generated by the two elements, and the embedding to $\Gamma$ is quasi-isometric.

Let $\alpha, \beta$ be quasi-axes of $a, b$ and set $D=\left|\alpha \cap_{\Lambda} \beta\right|$. Without loss of generality, we may assume that $\operatorname{tr}(b) \leq \operatorname{tr}(a)$. By Lemma 15, $D<\infty$.

Step 1. If $\frac{\operatorname{tr}(b)}{\operatorname{tr}(a)}>\frac{N \overline{17}}{M}$, then the claim is true.

Set $q=\frac{M}{N \sqrt{17}}$, and apply Proposition 17 to $a, b$. If $n \geq N$, and $m \geq q N[17=M$, then $\left\langle a^{n}, b^{m}\right\rangle$ is free and the embedding is quasi-isometric. Since $M>N[17$, we get the Claim.

In the rest, we may assume $\frac{\operatorname{tr}(b)}{\operatorname{tr}(a)} \leq \frac{N}{M}$. It follows that $10 K L P \operatorname{tr}(b) \leq \operatorname{tr}(a)$ since $\frac{N}{\overline{17}}<\frac{1}{10 K L P}$.

Step 2. If $M \operatorname{tr}(b)>D+26 \delta+14 \Delta+4 \Lambda+2$, then the claim is true.

Indeed, in this case, both $\operatorname{tr}\left(a^{n}\right), \operatorname{tr}\left(b^{m}\right)$ are $>D+26 \delta+14 \Delta+4 \Lambda+2$. (We use $\operatorname{tr}(b) \leq \operatorname{tr}(a)$.) Then by Proposition 8 , with the constant $U=1$, we get the Claim.

In the rest, we may also assume that $M \operatorname{tr}(b) \leq D+26 \delta+14 \Delta+4 \Lambda+2$. Since $10 K L N+100(\delta+\Delta+\Lambda+1) \leq M \operatorname{tr}(b)$ by the definition of $M$, it follows that $10 K L N+74(\delta+\Lambda+\Delta+1) \leq D$.

Step 3. It must be that $D \leq 2 \operatorname{tr}(a)$.

Assume $D>2 \operatorname{tr}(a)$. We will get a contradiction using the same idea as in the proof of Lemma [15] concerning the action of commutators $\left[b^{i}, a^{j}\right]$. Since $D \geq$ $74(\delta+\Lambda+\Delta+1)$ from Step 2, the set $\alpha \cap_{\Lambda} \beta$ looks like a narrow tube. Therefore, it makes sense to talk about the directions of the action by $a$ and $b$ along this tube. In the following, we assume that the actions by $a, b$ have the same direction along $\alpha \cap_{\Lambda} \beta$; otherwise, we consider $b^{-1}$ instead of $b$.

Let $p, p^{\prime} \in \alpha$ be the extremal points contained in $\alpha \cap_{\Lambda} \beta$, and set $\ell=\left[p, p^{\prime}\right]$. We know $|\ell| \geq D-2 \Lambda$. Since $D \geq 74(\delta+\Delta+\Lambda+1)$ from Step 2, it follows that $|\ell| \geq \frac{9}{10} D$.

Without loss of generality, we assume that $a$ moves $p$ toward $p^{\prime}$; i.e., $a(p)$, $a^{2}(p), \cdots$, are contained in the subpath of $\alpha$ between $p$ and $p^{\prime}$ until they go beyond $p^{\prime}$ (otherwise exchange $p$ and $p^{\prime}$ ). Since $D>2 \operatorname{tr}(a)$, at least $a(p)$ is contained in the subpath (use Lemma 2). Let $a^{J}(p)$ be the point which is closest (if there is more than one, then choose one of them) to the mid-point of the subpath; then $1 \leq J$. Note that $a^{J}(p)$ is far (compared to $\delta+\Delta+\Lambda$ ) from $p$ and $p^{\prime}$.

We claim that for all $1 \leq i \leq P K L$, we have $d\left(p,\left[b^{i}, a^{J}\right](p)\right) \leq 28 \delta+30 \Delta+12 \Lambda$. The argument is the same as the one for Lemma [14. We only need to verify that $\ell$ is long enough to apply the argument such that if we apply $a^{J}, b^{i}, a^{-J}$, then $b^{-i}$ to $p$, the point moves in a certain neighborhood of $\ell$ for all $1 \leq i \leq P K L$. We do not repeat the details.

Let $q \in \alpha$ be the point between $p$ and $p^{\prime}$ with $L=L(28 \delta+30 \Delta+12 \Lambda)=d(p, q)$. (Quasi-axes are continuous paths in this paper.) Such a point $q$ exists since $L \leq \frac{D}{10}$ 
by Step 2. By the same reason as in the case of $p$, we have $d\left(q,\left[b^{i}, a^{J}\right](q)\right) \leq$ $28 \delta+30 \Delta+12 \Lambda$ for all $1 \leq i \leq P K L$. Again we are using $L \leq \frac{D}{10}$ so that concerned points move in a certain neighborhood of $\ell$; therefore the argument applies.

Now by the acylindricity, since $d(p, q)=L(28 \delta+30 \Delta+12 \Lambda)$, there must be $1 \leq I<I^{\prime} \leq P K L$ such that $\left[b^{I}, a^{J}\right]=\left[b^{I^{\prime}}, a^{J}\right]$; therefore $\left[b^{I-I^{\prime}}, a^{J}\right]=1$, which gives a contradiction. This is the end of Step 3.

Step 4 . Since $D \leq 2 \operatorname{tr}(a)$, we can apply Proposition 18 to $a, b$. Therefore, if $n \geq N$, then $\left\langle b, a^{n}\right\rangle$ is a free group freely generated by the two elements, and the embedding is quasi-isometric. Since $M>N\left[18\right.$, we have shown the Claim. Note that $\left\langle b^{m}, a^{n}\right\rangle$ is a subgroup of $\left\langle b, a^{n}\right\rangle$.

Theorem 23 applies to the action of a word-hyperbolic group $G$ on a Cayley graph. An element $g$ is hyperbolic if and only if its order is infinite ([8]).

Therefore we obtain

Corollary 24. If $G$ is a word-hyperbolic group, then there exists $M$ such that for any two elements $a, b \in G$ of infinite order, either the subgroup $\langle a, b\rangle$ is elementary (i.e. contains an infinite cyclic group as a finite index subgroup) or else for any $n, m \geq M,\left\langle a^{n}, b^{m}\right\rangle$ is free and quasi-convex in $G$.

It seems this result is new (see [8, 8.2 E] for the statement without a bound on $n, m$; also the definition of quasi-convex subgroups).

Remark 25. It is more difficult to deal with the normal subgroup generated by $a^{n}, b^{m}$, or even just by $a^{n}$ (see Question 11 [14]). See the work of Delzant [5].

Remark 26. Theorem 23 regards two elements, but one can ask if there exists a constant $M$ such that if $a, b, c \in G$ are hyperbolic elements with certain condition (for example, pairwise independence), then $\left\langle a^{\ell}, b^{m}, c^{n}\right\rangle$ is free for any $\ell, m, n \geq M$. We remark that the rank of the free subgroup may not be three. Take two hyperbolic elements $a, b \in G$ which satisfy the commutator assumption in Theorem 23 (i.e., independent). For any $M>0$, set $c=a^{M} b a^{-M}$. Then, the pairs $a, c$ and $b, c$ are also independent, but $\left\langle a^{M}, b^{M}, c^{M}\right\rangle$ is equal to $\left\langle a^{M}, b^{M}\right\rangle$.

\section{Application to mapping Class group}

We discuss mapping class groups in this section. We apply results from Section 3 to pseudo-Anosov elements. Theorems 27 and 28 are the main results of the paper.

4.1. Uniform estimate. We apply Theorem 23 to the mapping class group, $\operatorname{Mod}(S)$, of a compact orientable surface $S$. Let $\mathcal{C}(S)$ be the curve graph of $S$ (see for example [13], 19] for the definition). Masur-Minsky [19] showed that $\mathcal{C}(S)$ is $\delta$-hyperbolic and an element $a \in \operatorname{Mod}(S)$ is pseudo-Anosov if and only if it acts as a hyperbolic isometry on $\mathcal{C}(S)$, and moreover (3]) there always exists an axis for $a^{N}$ if $N$ is large enough. Bowditch $[3$ showed that the action is acylindrical. (The curve graph is defined for a non-orientable surface and it is $\delta$-hyperbolic [2], but it seems it is unknown if the action by Mod is acylindrical.)

For a subgroup $G<\operatorname{Mod}(S)$, Farb-Mosher [6] introduced the notion of convexcocompact. It has been shown ([9, [15]) that $G$ is convex-cocompact if and only if for a point $c \in \mathcal{C}(S)$, the map from $G$ to $\mathcal{C}(S)$ sending $g$ to $g(c)$, namely the embedding by an orbit, is quasi-isometric. 
The following is an immediate consequence of Theorem 23. Apply it to the action of $\operatorname{Mod}(S)$ to $\mathcal{C}(S)$. Two pseudo-Anosov elements $a, b$ are called independent if $\left[a^{n}, b^{m}\right] \neq 1$ for any $n, m \neq 0$ (cf. [13]).

Theorem 27. Let $S$ be a compact orientable surface, and $\operatorname{Mod}(S)$ its mapping class group. Then there exists a constant $M(S)$ with the following property. Suppose $a, b \in \operatorname{Mod}(S)$ are pseudo-Anosov elements such that $\left[a^{n}, b^{m}\right] \neq 1$ for any $n, m \neq 0$. Then for any $n, m \geq M,\left\langle a^{n}, b^{m}\right\rangle$ is a free group freely generated by $a^{n}, b^{m}$ and is convex-cocompact. In particular all non-trivial elements in $\left\langle a^{n}, b^{m}\right\rangle$ are pseudoAnosov.

4.2. Non-uniform estimate and example. Let $a, b \in \operatorname{Mod}(S)$ be two independent pseudo-Anosov elements. It would be interesting to know for which $(n, m)$, $\left\langle a^{n}, b^{m}\right\rangle$ is a free group of rank two and convex-cocompact. The following theorem says that it is the case except for finitely many $(n, m)$. We do not know if the number of the exceptional pairs is bounded.

Theorem 28. Let $S$ be a compact orientable surface and $a, b$ two independent pseudo-Anosov elements. Then there exists $N \overline{28}=N$, which depends on a, $b$ such that for any $n \geq N$, both $\left\langle a, b^{n}\right\rangle$ and $\left\langle b, a^{n}\right\rangle$ are free groups freely generated by $a, b^{n}$ and $b, a^{n}$, respectively, and are convex-cocompact. In particular, $\left\langle a^{n}, b^{m}\right\rangle$ is a free group freely generated by the two elements and is convex-cocompact if $|n|+|m| \geq 2 N$ and $n m \neq 0$.

Proof. Set $N=N_{222}$. Apply Proposition 22 to $a, b$ for the action on $\mathcal{C}(S)$.

The constant $N \overline{28}$ must depend on $a, b$ as the following example shows.

Example 29. Let $S$ be a compact orientable surface which is not a sphere with less than four punctures or a torus. If $n>0$ is sufficiently large, then there exist two independent pseudo-Anosov elements $f, g \in \operatorname{Mod}(S)$ such that $\left\langle g, f^{n}\right\rangle$ is not a free group.

To see this, take $f, a \in \operatorname{Mod}(S)$ such that $f$ is pseudo-Anosov, $a$ is non-trivial torsion and $\langle f, a\rangle$ is not virtually cyclic. To find such $a, f$, first take a non-trivial torsion element $a \in \operatorname{Mod}(S)$ such that there is a non-trivial and non-peripheral simple closed curve $\sigma$ on $S$ which is not homotopic to $a(\sigma)$. One can find such $a$ easily. Then one can find a desired $f$. For example, take any pseudo-Anosov element $h$ on $S$. Let $d$ be a Dehn-twist along $\sigma$. Set $f=d^{m} h d^{-m}$. We choose a sufficiently large $m>0$ later. It is clear that $f$ is pseudo-Anosov, and the two laminations which are invariant by $f$, which we regard as a set of two points, fix $(f)$, in the boundary of the Teichmüller space of $S$, must be moved by a (i.e. $\operatorname{fix}(f) \cap a(\operatorname{fix}(f))=\emptyset)$ if $m$ is sufficiently large. For such $m$, it follows by a standard argument that $\langle f, a\rangle$ is not virtually cyclic (cf. [12]).

Now, for sufficiently large $n, f^{n} a$ is pseudo-Anosov and independent from $f$. One can show this using the curve graph of $S, \mathcal{C}(S)$, which is $\delta$-hyperbolic. If necessary, replace $f$ by some power of it in advance and assume that $f$ leaves a geodesic $\gamma$ in $\mathcal{C}(S)$ invariant. By our assumption $\gamma \cap_{\Lambda} a(\gamma)$ is bounded; otherwise $\langle f, a\rangle$ would be virtually cyclic. For each $n>0$, one can find a line which is invariant by $f^{n} a$ using a piece of $\gamma$, a fundamental domain for the action of $f^{n}$, and the action of $a$. Then, for sufficiently large $n$, using $\delta$-hyperbolic geometry of $\mathcal{C}(S)$, one can show that the line is indeed a quasi-geodesic; therefore $f^{n} a$ is pseudo-Anosov. Moreover, 
for sufficiently large $n$, the quasi-geodesic has two points at infinity of $\mathcal{C}(S)$ which are disjoint from the two points for $\gamma$. It implies that $f^{n} a$ and $f$ are independent. Set $g=f^{n} a$. Then $\left\langle g, f^{n}\right\rangle$ is not free since it contains the torsion element $a$.

\section{Appendix (Proof of Lemmas 1 and 21)}

We will use Lemma 4 repeatedly. Moreover, we use a refined version such that if $[A, B],[C, D]$ are geodesics in $\delta$-hyperbolic space with $|A-C|,|B-D| \leq L$ and, moreover, if $[A, B]$ is much longer than $L, \delta$, then most of $[A, B]$ except for the part near $A$ and $B$ (whose length is at most $L+10 \delta$ ) is in the $2 \delta$-neighborhood of $[C, D]$. (The argument is the same. We leave the argument to the reader.) Typically, we apply this to points around the mid-point of $[A, B]$.

Proof of Lemma 1. We will show the lemma for $\Delta=418 \delta$.

If $\delta=0$, then $\Gamma$ is a tree; therefore an axis exists for a hyperbolic isometry, 22. We assume that $\delta>0$.

Since $a$ is hyperbolic, we can choose $N$ such that $\operatorname{tr}\left(a^{N}\right) \geq 1000 \delta$. Set $A=a^{N}$. Let $x \in \Gamma$ be any point and let $m$ be the mid-point of $[x, A(x)]$.

Define a path (parameterized by the arc length) by

$$
\gamma=\sum_{n \in \mathbb{Z}} a^{n}([m, a(m)]) .
$$

Main claim. There is a constant $\Delta$, which depends only on $\delta$, such that for any points $p, q \in \gamma$ :

(1) $[p, q] \subset N_{\Delta}\left(\gamma_{p, q}\right)$.

(2) $\gamma_{p, q} \subset N_{\Delta}([p, q])$.

Clearly, $a(\gamma)=\gamma$ and $\gamma$ is a desired path for the lemma with $L=|m-a(m)|$. We will find a bound $\Delta \leq 418 \delta$. As we said, there is more than one possibility for $\gamma_{p, q}$ depending on the parameters for $p, q$, but the main claim holds for any of them. Therefore any two of them are in the $2 \Delta$-neighborhood of each other.

The map $\gamma$ is not injective in general. If we can choose $m$ such that $\gamma$ is injective, then some of the arguments in this paper become less complicated. For example, $\gamma_{p, q}$ will be uniquely defined. We leave it as a question if an injective quasi-axis exists or not.

We start the proof of the main claim. We define another path by

$$
\alpha=\sum_{i \in \mathbb{Z}} A^{i}([m, A(m)]) .
$$

(This $\alpha$ has nothing to do with the $\alpha$ in Lemma 1) Clearly $\alpha=A(\alpha)$. We show

Claim 1. For each $0 \leq i$,

$$
m, A(m), A^{2}(m), \cdots, A^{i}(m) \in N_{2 \delta}\left(\left[m, A^{i}(m)\right]\right) .
$$

The argument is elementary and standard by drawing triangles and geodesics. Let $s \in[m, A(m)]$ be a point such that there exist $t \in[m, A(x)], u \in[A(x), A(m)]$ with $|t-s| \leq \delta,|u-s| \leq \delta$. Such points exist since the triangle $(m, A(x), A(m))$ is $\delta$-thin. Notice that $s$ is around the mid-point of $[m, A(m)]$ (at most, say, $10 \delta$ away), since $|m-A(x)|=|A(x)-A(m)|$; therefore, $|u-A(m)|,|m-t|$ are long compared to $\delta$ (in fact $\geq 490 \delta$ ). 
We argue by induction on $i$. For $i=1$, there is nothing to prove and assume $i=2$. Since the triangle $\left(m, A(m), A^{2}(m)\right)$ is $\delta$-thin, there are $y, z \in\left[m, A^{2}(m)\right]$ with $|s-y|,|A(s)-z| \leq \delta$ (use that $s, A(s)$ are far from $A(m)$ ). On the other hand, $A(m)$ is around the mid-point of the long geodesic $[u, A(t)]$, which is in the $4 \delta$ neighborhood of the geodesic $[y, z] \subset\left[m, A^{2}(m)\right]$ by Lemma 4 . Since $A(m)$ is around the mid-point of $[u, A(t)]$, there must be a point $v \in[y, z]$ with $|v-A(m)| \leq 2 \delta$ (see the remark in the beginning of this section). We finish the case with $i=2$ (see Figure 5).

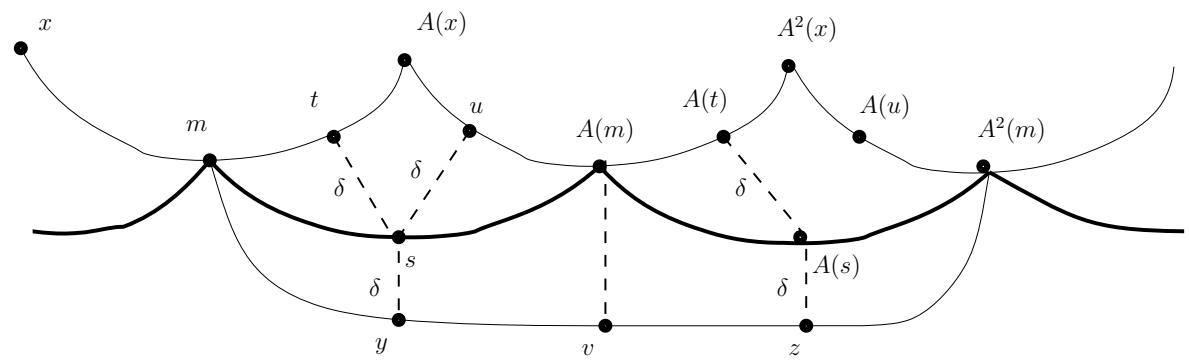

Figure 5. $|A(m)-v| \leq 4 \delta$ by Lemma 4. In fact, $\leq 2 \delta$.

Now assume the claim is true up to $i$. First, notice that we also have

$$
s, A(s), \cdots, A^{i-1}(s) \in N_{2 \delta}\left(\left[m, A^{i}(m)\right]\right) .
$$

This is because each $A^{j}(s)$ is around the mid-point of a long geodesic $\left[A^{j}(m)\right.$, $\left.A^{j+1}(m)\right]$ whose end points are in the $2 \delta$-neighborhood of $\left[m, A^{i}(m)\right]$. Let $r_{j} \in$ $\left[m, A^{i}(m)\right]$ with $\left|r_{j}-A^{j}(s)\right| \leq 2 \delta$ for $0 \leq j \leq i-1$. By the $\delta$-thinness of the triangle $\left(m, A^{i}(m), A^{i+1}(m)\right)$, let $q_{i} \in\left[m, A^{i+1}(m)\right]$ be a point with $\left|q_{i}-r_{i}\right| \leq \delta$ for $0 \leq j \leq i-1$.

Now, $A^{j}(m)(1 \leq j \leq i-1)$ is on $\left[A^{j-1}(u), A^{j}(t)\right]$ around its mid-point. The end points of this geodesic and the end points of the geodesic $\left[q_{j-1}, q_{j}\right]$ are at most $4 \delta$ from each other (for this estimate, use $A^{j-1}(s), r_{j-1}$ and $A^{j}(s), r_{j}$ to interpolate), so $A^{j}(m)$ must be contained in the $2 \delta$-neighborhood of $\left[q_{j-1}, q_{j}\right] \subset\left[m, A^{i+1}(m)\right]$. We are left with $A^{i}(m)$. For that, choose $q_{i} \in\left[m, A^{i+1}(m)\right]$ with $\left|A^{i}(s)-q_{i}\right| \leq \delta$ and argue in the same way. The induction is complete and Claim 1 is shown.

From Claim 1, we have the following.

Claim 2. For any $p=A^{i}(m), q=A^{j}(m) \in \alpha,(i<j)$, we have

(1) $[p, q] \subset N_{4 \delta}\left(\alpha_{p, q}\right)$.

(2) $\alpha_{p, q} \subset N_{4 \delta}([p, q])$.

Here, $\alpha_{p, q}$ means a specific subpath (the most natural one) between $p, q$ such that the parameters for $p=A^{i}(m), q=A^{j}(m)$ are $i L, j L$ if $m=\alpha(0)$, where $L=|m-A(m)|$.

We may assume $i=0$. Using Claim 1 , let $p_{k} \in[p, q]$ with $\left|p_{k}-A^{k}(m)\right| \leq 2 \delta$. Then for each $k,\left[p_{k}, p_{k+1}\right]$ and $\left[A^{k}(m), A^{k+1}(m)\right]$ are in the $4 \delta$-neighborhood of each other and Claim 2 is proved. 
In general, $\alpha$ is not $a$-invariant, but we show

Claim 3. For any $n$,

$$
a^{n}(m) \in N_{8 \delta}(\alpha) .
$$

First, since $\alpha$ is invariant by $a^{N}$, there exists some constant $L$ such that for any $n, a^{n}(\alpha)$ and $\alpha$ are in the $L$-neighborhood of each other.

For a given $n$, we choose points $p=A^{i}(m), q=A^{j}(m) \in \alpha$ such that $m$ is around the mid-point of $[p, q]$ and $|p-q|$ is much larger than $\left|m-a^{n}(m)\right|$ and $L$. Since $m \in N_{2 \delta}([p, q])$ by Claim 1 , we have $a^{n}(m) \in N_{2 \delta}\left(a^{n}([p, q])\right)$. Also, since $\left|p-a^{n}(p)\right|=\left|m-a^{n}(m)\right|=\left|q-a^{n}(q)\right|$ (they are on an orbit by $A$ ), most of $a^{n}([p, q])$, except near the end points (whose length is comparable to $\left|m-a^{n}(m)\right|$ and $\delta$, while $|p-q|$ is much larger) is contained in $N_{2 \delta}([p, q])$. It follows that in fact $a^{n}(m) \in N_{4 \delta}([p, q]) \subset N_{8 \delta}(\alpha)$. The last inclusion is by Claim 2. Claim 3 is shown.

Let $I>0$ be an integer, and consider points $m, a(m), a^{2}(m), \cdots, a^{I}(m)$. By Claims 2 and 3, there is a (very long but finite) geodesic $\rho$ such that all of those points are not only in the $(4+8) \delta$-neighborhood of $\rho$, but are also very far from the end points of $\rho$, and $\rho$ and $a^{i}(\rho)(0 \leq i \leq I)$ are in the $2 \delta$-neighborhood of each other except near the end points (this exceptional part is much shorter than $\rho$ ). In fact we can take two points $p, q \in \alpha$ and take the geodesic $[p, q]=\rho$. Set $K=12 \delta$; then we have

$$
m, a(m), \cdots, a^{I}(m) \in N_{K}(\rho) .
$$

Let $<$ denote a natural linear order on $\rho$. There are two, and we specify them soon. Since $a^{i}(\rho), 0 \leq i \leq I$ are in the $2 \delta$-neighborhood of each other for the most part (except for the part near the end points), we can put an order $<$ on each of them so that they are compatible. Then $a$ maps $\rho$ to $a(\rho)$ preserving the order. If $\operatorname{tr}\left(a^{i}\right), i>0$, is large enough compared to $2 \delta$, it makes sense to say $a^{i}$ maps $\rho$ toward positive or negative w.r.t. the order, and it does not depend on $i$. We choose the order such that $a^{i}$ maps $\rho$ to the positive direction. Also, each $a^{i}(\rho)$ is two-ended, and we can naturally identify the ends of two geodesics, and $a$ preserves each end.

Let $p_{i} \in \rho$ be a point with $\left|p_{i}-a^{i}(m)\right| \leq K$.

Claim 4. If $p_{i}<p_{0}$ for some $i>0$, then $\left|p_{i}-p_{0}\right| \leq L=2 K+12 \delta$.

To argue by contradiction, assume $p_{i}<p_{0}$ and $\left|p_{i}-p_{0}\right|>L$. Although $p_{i}<p_{0}$ may depend on the choice of $\rho$ in general, by $\left|p_{i}-p_{0}\right|>L$, it does not depend on the choice of $\rho$ since they are in the $2 \delta$-neighborhood of each other. By changing $\rho,\left|p_{i}-p_{0}\right|$ may change at most $4 \delta$. We will take $I$ larger and $\rho$ longer if needed, and always have $\left|p_{i}-p_{0}\right|>L-4 \delta=2 K+8 \delta$.

Set $x=p_{0} \in \rho$; then $|x-m| \leq K$. By our assumption, $\left|a^{i}(x)-x\right|>L-4 \delta-2 K$. Since $a^{i}(x) \in a^{i}(\rho)$, let $x_{i} \in \rho$ be a point with $\left|a^{i}(x)-x_{i}\right| \leq 2 \delta$. Then $\left|x-x_{i}\right|>$ $L-4 \delta-2 K-2 \delta$ and $x_{i}<x$ since $p_{i}<p_{0}=x$. (Since $L-2 K-6 \delta \geq 4 \delta$, whether $x_{i}<x$ or not does not depend on the choice of $\rho$.)

For the point $a^{2 i}(x) \in a^{2 i}(\rho)$, let $x_{2 i} \in \rho$ be a point with $\left|x_{2 i}-a^{2 i}(x)\right| \leq 2 \delta$. Then $\left|x_{i}-x_{2 i}\right|>L-2 K-8 \delta$, and $x_{2 i}<x_{i}$ on $\rho$. Again, this makes sense since $L-2 K-8 \delta \geq 4 \delta$. We continue in the same way as long as possible; namely, let $x_{k i} \in a^{k i}(\rho)$ be a point with $\left|a^{k i}(x)-x_{k i}\right| \leq 2 \delta$. Then, $\left|x_{k i}-x_{(k+1) i}\right| \geq L-2 K-8 \delta$, and $x_{(k+1) i}<x_{k i}$ on $\rho$. 
In this way, we obtain a sequence (see Figure 6)

$$
x>x_{i}>x_{2 i}>x_{3 i}>\cdots>x_{k i}
$$

if $k i<I$ (in other words, as long as $a^{k i}(x)$ is in the $2 \delta$-neighborhood of $\rho$ ).

This argument applies to any $I>0$. But this will give a contradiction since it means that $a^{k i}$ ( $k$ sufficiently large) maps $\rho$ to the negative direction in terms of the order <. On the other hand, for example, by the choice of $N, a^{N}$ maps $\rho$ to the positive direction. Claim 4 is shown.

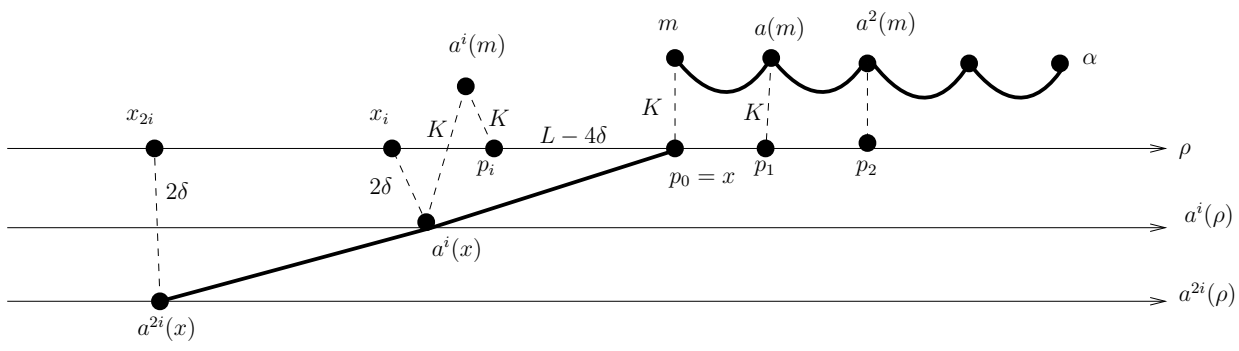

Figure 6. Points on $\rho: x>x_{i}>x_{2 i}>x_{3 i}>\cdots>x_{k i}$

We already know $[m, a(m)] \subset N_{K+2 \delta}(\rho)$.

Claim 5. Suppose $x \in[m, a(m)]$. Let $p_{0}, p_{1}, p_{x} \in \rho$ be the nearest points for $m, a(m), x$. If $p_{x}<p_{0}$, then $\left|p_{x}-p_{0}\right| \leq L+10(K+2 \delta)$. Also, if $p_{1}<p_{x}$, then $\left|p_{x}-p_{1}\right| \leq L+10(K+2 \delta)$

We only prove the first assertion since the second one is the same by changing the role of $a$ and $a^{-1}$. Indeed, assume $p_{x}<p_{0}$ and $\left|p_{x}-p_{0}\right|>L+10(K+2 \delta)$. Let $P<p_{0}$ be the point on $\rho$ with $\left|P-p_{0}\right|=L$. By Claim $4, p_{x}<P \leq p_{1}$. It follows that there are points $w \in[m, x]$ and $y \in[x, a(m)]$ such that their nearest points on $\rho, p_{w}, p_{y}$ satisfy $\left|p_{w}-P\right| \leq 2(K+2 \delta),\left|p_{y}-P\right| \leq 2(K+2 \delta)$ (the nearest projection is not continuous, but at most $2(K+2 \delta)$ gap). This implies that $|w-y| \leq 6(K+2 \delta)$. But we have $w<x<y$; therefore $\left|p_{x}-p_{y}\right| \leq 8(K+2 \delta)$, so that $\left|p_{x}-P\right| \leq 10(K+2 \delta)$, which implies that $\left|p_{x}-p_{0}\right| \leq L+10(K+2 \delta)$, a contradiction. Claim 5 is shown (see Figure 7).

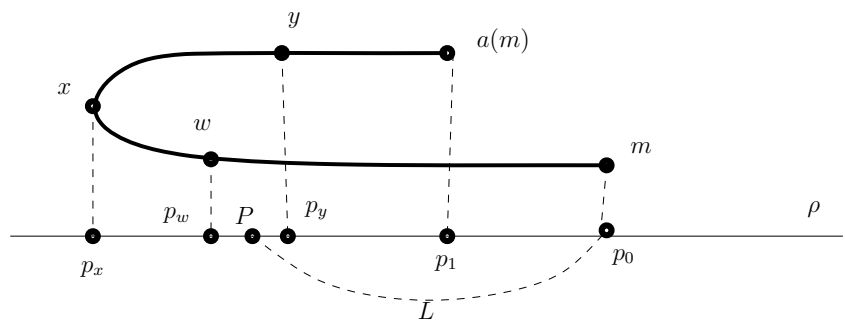

FiguRE 7. Bound on the backtrack 
We start proving the main claim. Suppose $x=\gamma(u), y=\gamma(v)$ with $u<v$. We claim that if the nearest points project to $\rho, p_{x}, p_{y}$, satisfy $p_{x}>p_{y}$, then $\left|p_{x}-p_{y}\right| \leq 3 L+20(K+2 \delta)$. Namely, a backtrack is bounded.

Indeed, assume $x$ is contained in the subpath, say, $[m, a(m)]$, and $y$ is contained in $\left[a^{n}(m), a^{n+1}(m)\right], n>0$. Then at worst, it can go back from $p_{x}$ to $p_{1}$ by $L+$ $10(K+2 \delta)$ (Claim 5 ), then by $L$ from $p_{1}$ to $p_{n}$ (use Claim 4 ), then by $L+10(K+2 \delta)$ (Claim 5) from $p_{n}$ to $p_{y}$, which adds up to $3 L+20(K+2 \delta)=Q$. The claim is proved.

It follows that $\gamma_{x, y} \subset N_{Q+K+2 \delta}\left(\left[p_{x}, p_{y}\right]\right)$. On the other hand, $\left[p_{x}, p_{y}\right] \subset N_{2(K+2 \delta)}$ $\left(\gamma_{x, y}\right)$ (the map from $\gamma$ to $\rho$ is not continuous, but the diameter of the gap is at most $2(K+2 \delta))$. Therefore, they are in the $(Q+K+2 \delta)$-neighborhood of each other. (We said that there is an ambiguity for $\gamma_{x, y}$ concerning the parameters for $x$ and $y$, but the above inclusions hold for any choice of the parameters. Therefore the lemma will also hold for any subpath.)

Now, $[x, y]$ and $\left[p_{x}, p_{y}\right]$ are in the $(K+4 \delta)$ - neighborhood of each other; therefore, $\gamma_{x, y}$ and $[x, y]$ are in the $(Q+K+2 \delta+K+4 \delta)$-neighborhood of each other. The main claim is shown for $\Delta=Q+2 K+6 \delta=418 \delta$, and the proof of Lemma 1 is complete.

Proof of Lemma 2, The second inequality is clear from the definition of $\operatorname{tr}(a)$. For the first inequality, we first show that

Claim. If $a$ is hyperbolic and $\alpha$ is a quasi-axis, then for each point $p \in \alpha$ and any $n$, we have

$$
\left|p-a^{n}(p)\right| \geq n(|p-a(p)|-2 \delta-2 \Delta) .
$$

Indeed, there is nothing to prove if $|p-a(p)| \leq 2 \delta+2 \Delta$. Assume $U=$ $|p-a(p)|-2 \delta-2 \Delta>0$. Since $p, a(p), a^{2}(p)$ are on the quasi-axis $\alpha$, the point $a(p)$ is at most at $\Delta$ from some point $q \in\left[p, a^{2}(p)\right]$. Then by the triangle inequality, we have $\left|p-a^{2}(p)\right| \geq|p-a(p)|+\left|a(p)-a^{2}(p)\right|-2 \Delta$. This means that the three points condition is satisfied by the sequence $\left\{a^{n}(p)\right\}_{n}$ and $U$. Now, by Proposition 7 , we have the desired inequality, and the claim is shown.

Fix $N \geq 1$, and apply the claim to the element $a^{N}$ and $\alpha$. Then for each $n$, $\left|p-a^{N n}(p)\right| \geq n\left(\left|p-a^{N}(p)\right|-2 \delta-2 \Delta\right)$. So,

$$
\left|p-a^{N n}(p)\right| / N n \geq\left(\left|p-a^{N}(p)\right|-2 \delta-2 \Delta\right) / N .
$$

Letting $n \rightarrow \infty$, we have $\operatorname{tr}(a) \geq\left(\left|p-a^{N}(p)\right|-2 \delta-2 \Delta\right) / N$.

\section{ACKNOWLEDGEMENTS}

The author would like to thank Z. Sela for many insightful suggestions, and L. Mosher for his interest and comments. He is grateful to M. Bestvina and T. Delzant. He thanks the patient referee, who helped to improve the presentation of the paper. The work was started during the author's stay at MSRI in Fall 2007. A part of it was done at the Institut Mittag-Leffler (Djursholm, Sweden) and Max Planck Institute for Mathematics in Bonn. He appreciates their hospitality. The author was partially supported by Grant-in-Aid for Scientific Research (No. 60229078, No. 23244005). 


\section{REFERENCES}

[1] Mladen Bestvina and Koji Fujiwara, Bounded cohomology of subgroups of mapping class groups, Geom. Topol. 6 (2002), 69-89 (electronic), DOI 10.2140/gt.2002.6.69. MR.1914565 (2003f:57003)

[2] Mladen Bestvina and Koji Fujiwara, Quasi-homomorphisms on mapping class groups, Glas. Mat. Ser. III 42(62) (2007), no. 1, 213-236, DOI 10.3336/gm.42.1.15. MR2332668 (2008k:57002)

[3] Brian H. Bowditch, Tight geodesics in the curve complex, Invent. Math. 171 (2008), no. 2, 281-300, DOI 10.1007/s00222-007-0081-y. MR2367021(2008m:57040)

[4] Thomas Delzant, Sous-groupes à deux générateurs des groupes hyperboliques (French), Group theory from a geometrical viewpoint (Trieste, 1990), World Sci. Publ., River Edge, NJ, 1991, pp. 177-189. MR1170366 (93c:57001)

[5] Thomas Delzant, Sous-groupes distingués et quotients des groupes hyperboliques (French), Duke Math. J. 83 (1996), no. 3, 661-682, DOI 10.1215/S0012-7094-96-08321-0. MR1390660 (97d:20041)

[6] Benson Farb and Lee Mosher, Convex cocompact subgroups of mapping class groups, Geom. Topol. 6 (2002), 91-152 (electronic), DOI 10.2140/gt.2002.6.91. MR.1914566 (2003i:20069)

[7] Koji Fujiwara, Subgroups generated by two pseudo-Anosov elements in a mapping class group. I. Uniform exponential growth, Groups of diffeomorphisms, Adv. Stud. Pure Math., vol. 52, Math. Soc. Japan, Tokyo, 2008, pp. 283-296. MR2509713 (2010j:20063)

[8] M. Gromov, Hyperbolic groups, Essays in group theory, Math. Sci. Res. Inst. Publ., vol. 8, Springer, New York, 1987, pp. 75-263, DOI 10.1007/978-1-4613-9586-7_3. MR919829 (89e:20070)

[9] U. Hamenstädt, Word hyperbolic extensions of surface groups, eprint 2005, arXiv:math/0505244.

[10] Hessam Hamidi-Tehrani, Groups generated by positive multi-twists and the fake lantern problem, Algebr. Geom. Topol. 2 (2002), 1155-1178 (electronic), DOI 10.2140/agt.2002.2.1155. MR $1943336(2003 \mathrm{j}: 57002)$

[11] Atsushi Ishida, The structure of subgroup of mapping class groups generated by two Dehn twists, Proc. Japan Acad. Ser. A Math. Sci. 72 (1996), no. 10, 240-241. MR 1435728 (97m:57015)

[12] Nikolai V. Ivanov, Subgroups of Teichmüller modular groups, Translations of Mathematical Monographs, vol. 115, American Mathematical Society, Providence, RI, 1992. Translated from the Russian by E. J. F. Primrose and revised by the author. MR 1195787 (93k:57031)

[13] Nikolai V. Ivanov, Mapping class groups, Handbook of geometric topology, North-Holland, Amsterdam, 2002, pp. 523-633. MR 1886678(2003h:57022)

[14] Nikolai V. Ivanov, Fifteen problems about the mapping class groups, Problems on mapping class groups and related topics, Proc. Sympos. Pure Math., vol. 74, Amer. Math. Soc., Providence, RI, 2006, pp. 71-80, DOI 10.1090/pspum/074/2264532. MR2264532(2008b:57003)

[15] Richard P. Kent IV and Christopher J. Leininger, Shadows of mapping class groups: capturing convex cocompactness, Geom. Funct. Anal. 18 (2008), no. 4, 1270-1325, DOI 10.1007/s00039008-0680-9. MR2465691(2009j:20056)

[16] Malik Koubi, Croissance uniforme dans les groupes hyperboliques (French, with English and French summaries), Ann. Inst. Fourier (Grenoble) 48 (1998), no. 5, 1441-1453. MR.1662255 (99m:20080)

[17] Christopher J. Leininger and Dan Margalit, Two-generator subgroups of the pure braid group, Geom. Dedicata 147 (2010), 107-113, DOI 10.1007/s10711-009-9440-8. MR2660568 (2011d:20074)

[18] Johanna Mangahas, Uniform uniform exponential growth of subgroups of the mapping class group, Geom. Funct. Anal. 19 (2010), no. 5, 1468-1480, DOI 10.1007/s00039-009-0038-y. MR 2585580(2011d:57002)

[19] Howard A. Masur and Yair N. Minsky, Geometry of the complex of curves. I. Hyperbolicity, Invent. Math. 138 (1999), no. 1, 103-149, DOI 10.1007/s002220050343. MR 1714338 (2000i:57027)

[20] John McCarthy, A "Tits-alternative" for subgroups of surface mapping class groups, Trans. Amer. Math. Soc. 291 (1985), no. 2, 583-612, DOI 10.2307/2000100. MR800253 (87f:57011) 
[21] George Pólya and Gabor Szego, Problems and theorems in analysis. I, Series, integral calculus, theory of functions; Classics in Mathematics, Springer-Verlag, Berlin, 1998. Translated from the German by Dorothee Aeppli; Reprint of the 1978 English translation. MR 1492447

[22] Jean-Pierre Serre, Trees, Springer-Verlag, Berlin-New York, 1980. Translated from the French by John Stillwell. MR607504 (82c:20083)

Department of Mathematics, Kyoto University, Kyoto, 606-8502, Japan

E-mail address: kfujiwara@math.kyoto-u.ac.jp 